Review

\title{
Research Trends on the Application of Electrolyzed Water in Food Preservation and Sanitation
}

\author{
Pianpian Yan, Ramachandran Chelliah (D, Kyoung hee Jo and Deog Hwan Oh *(D) \\ Department of Food Science and Biotechnology, College of Agriculture and Life Science, Kangwon National \\ University, Chuncheon 24341, Korea; pianpianyan1@gmail.com (P.Y.); ramachandran865@gmail.com (R.C.); \\ ttt1528@naver.com (K.h.J.) \\ * Correspondence: deoghwa@kangwon.ac.kr; Tel.: +82-33-250-6457
}

check for updates

Citation: Yan, P.; Chelliah, R.; Jo, K.h.; Oh, D.H. Research Trends on the Application of Electrolyzed Water in Food Preservation and Sanitation. Processes 2021, 9, 2240. https:// doi.org/10.3390/pr9122240

Academic Editor:

Ángeles Alonso-Moraga

Received: 3 November 2021

Accepted: 9 December 2021

Published: 13 December 2021

Publisher's Note: MDPI stays neutral with regard to jurisdictional claims in published maps and institutional affiliations.

Copyright: (c) 2021 by the authors. Licensee MDPI, Basel, Switzerland. This article is an open access article distributed under the terms and conditions of the Creative Commons Attribution (CC BY) license (https:// creativecommons.org/licenses/by/ $4.0 /)$.

\begin{abstract}
Electrolyzed water (EW) has been proposed as a novel promising sanitizer and cleaner in recent years. It is an effective antimicrobial and antibiofilm agent that has several advantages of being on the spot, environmentally friendly, cheap, and safe for human beings. Therefore, EW has been applied widely in various fields, including agriculture, food sanitation, livestock management, medical disinfection, clinical, and other fields using antibacterial technology. Currently, EW has potential significance for high-risk settings in hospitals and other clinical facilities. The research focus has been shifted toward the application of slightly acidic EW as more effective with some supplemental chemical and physical treatment methods such as ultraviolet radiations and ultrasound. This review article summarizes the possible mechanism of action and highlights the latest research studies in antimicrobial applications.
\end{abstract}

Keywords: electrolyzed water; germicidal mechanisms; application; food industry; hurdle enhancement

\section{Introduction}

Foodborne disease refers to any illness caused by spoilage of contaminated food with pathogenic bacteria, parasites, or viruses [1,2]. Foodborne diseases take a significant toll on health [3] and are still not under control, and the epidemic may cause health concerns and economic losses [4]. The most common causes of foodborne illness are diarrheal disease agents, particularly Campylobacter spp. and norovirus [5]. However, there is no single clinical syndrome for all foodborne diseases [6]. The World Health Organization (WHO) estimates that there are nearly 1.7 billion cases of diarrheal disease in children each year, mainly caused by contaminated water and food. In addition, diarrheal disease caused 230,000 deaths cases and is mainly caused by non-typhoidal Salmonella enterica in 2010 [7]. Therefore, developing effective sanitizers for killing pathogens is one of the most critical food safety steps in agricultural and food products for the hazard analysis and critical control point (HACCP) principle.

Currently, various approaches have been used to eradicate foodborne pathogens while maximizing maintenance quality. Numerous commercial sanitizers, such as physical technology (ozone), chemical technology (chlorine compounds, peroxide mixtures, quaternary ammonium compounds), and biological technology (essential oil), have been used as disinfection techniques throughout the food chain [8-12]. However, some of these technologies in the food field are not entirely acceptable due to disadvantages such as chemical residues, limited inactivation, adverse effects on food quality, or potential toxicity to human beings or the environment. Thus, safe and effective disinfectants in the food industry are urgently needed.

Electrolyzed water $(\mathrm{EW})$ containing hypochlorous acid $(\mathrm{HOCl})$ has been proposed as a novel promising sanitizer and cleaner in recent years, as shown in the Figure 1. Sodium hypochlorite and EW are advanced chlorine-based sanitizers. The active compound of sodium hypochlorite solution is a chemical compound with the formula $\mathrm{NaOCl}$ that acted 
with carbon dioxide to produce hypochlorous acid in order to assure the safety of the products. However, EW is a solution produced by electrolyzing diluted electrolytes usually containing $\mathrm{NaCl}$ in an electrolysis cell, and the main microbial inactivation component is free chlorines species $[13,14]$. The electrolysis cell is the location where positively charged (anode) and negatively charged (cathode) electrodes were separated by a non-selective membrane [15]. EW comprises five types according to the final solution's $\mathrm{pH}$ value and has $\mathrm{pH}$ ranges including strongly acidic EW (StAEW) $(\mathrm{pH} 2.2-2.7)$, weakly acidic EW (WAEW) (pH 2.7-5.0), slightly acidic EW (SAEW) (pH 5.0-6.5), neutral EW (NEW) (pH 6.5-7.5), and alkaline EW(AkEW) (pH 11.0-13.8). EW showed antimicrobial activity against a wide range of microorganisms and eliminated the most common forms of bacteria, fungi, viruses, and spores in food, food processing surface, and non-food-contact surfaces in a relatively short time (usually within 10-20 s) [16-18]. Various studies have reported EW's antimicrobial effect in vegetables, fruits, poultry, and seafood [19-21]. Additionally, EW with available chlorine concentration was used instead of tap water during seed soaking and germination of seeds (mung bean, pea, brown rice, alfalfa, and buckwheat) [22-25]. This process resulted in inhibition of microbial growth, effects on morphological characteristics of sprouts, and even accumulation of functional components [26].

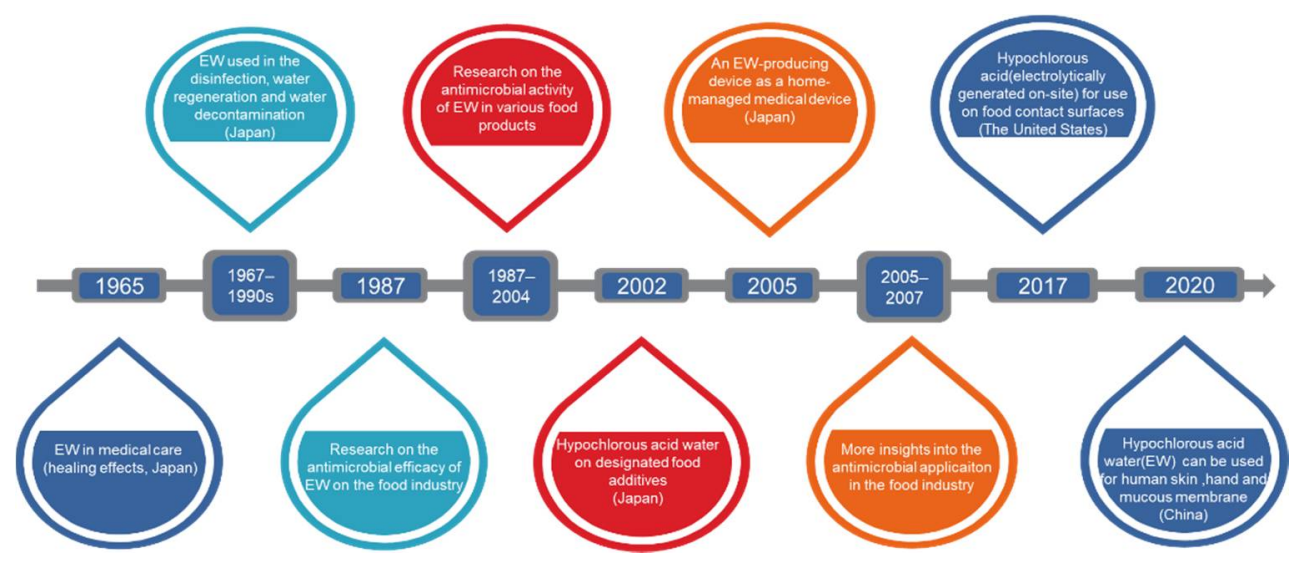

Figure 1. History of electrolyzed water applications.

The antimicrobial activity of EW is currently evaluated in the medical area, such as venous ulcers in the legs, diabetic foot ulcers, or feminine hygiene [14]. Scientists also pay more attention to the EW applied in the clinical field (medical sterilization) due to anti-infection and cell proliferative effect.

Hurdle technology was applied as an intelligent combination of two or more preservatives techniques at less severe levels [27]. However, some studies have demonstrated that EW's application is limited by factors such as organics materials (lipids, proteins, etc.) and corrosion of equipment in contact with acidic or alkaline EW [28]. Therefore, combining EW with other disinfection methods (physical and chemical technology) could effectively achieve desirable results [29]. This paper aims to review recent developments on EW application including agriculture, food sanitation, and livestock management, especially for germination and the clinical field. In addition, the potential germicidal mechanisms and hurdle technology were discussed.

\section{The Germicidal Mechanisms}

Underlying microbial inactivation by EW have not been completely elucidated. However, a model roughly explaining germicidal mechanisms of EW has been developed, as shown in Figure 2. Chlorine species $\left(\mathrm{HOCl}, \mathrm{Cl}_{2}\right.$, and $\left.\mathrm{OCl}^{-}\right)$play an essential role in the antimicrobial efficacy of EW [30]. Briefly, EW showed its antimicrobial activity by attacking various cellular targets (cytoderm, intracellular components, and outer membrane). Firstly, $\mathrm{HOCl}$ can penetrate the cell membrane's lipid bilayer by passive diffusion due to its electrical neutrality and molecular size [31]. Cheng et al. reported that the bacterial surface was 
changed from smooth, consecutive, and bright into rough, shrunken, and even lysed after EW treatment [32]. In addition, Ding et al. reported that SAEW disrupted cell membrane permeability by damaging membrane proteins, entering the cells, and causing the agglutination of cellular inclusions [33]. Meanwhile, EW intensified membrane permeability and decreased the activity of 2,3,5-triphenyl tetrazolium chloride (TTC)-dehydrogenase, which resulted in the leakage of $\mathrm{K}^{+}, \mathrm{DNA}$, and proteins [34].

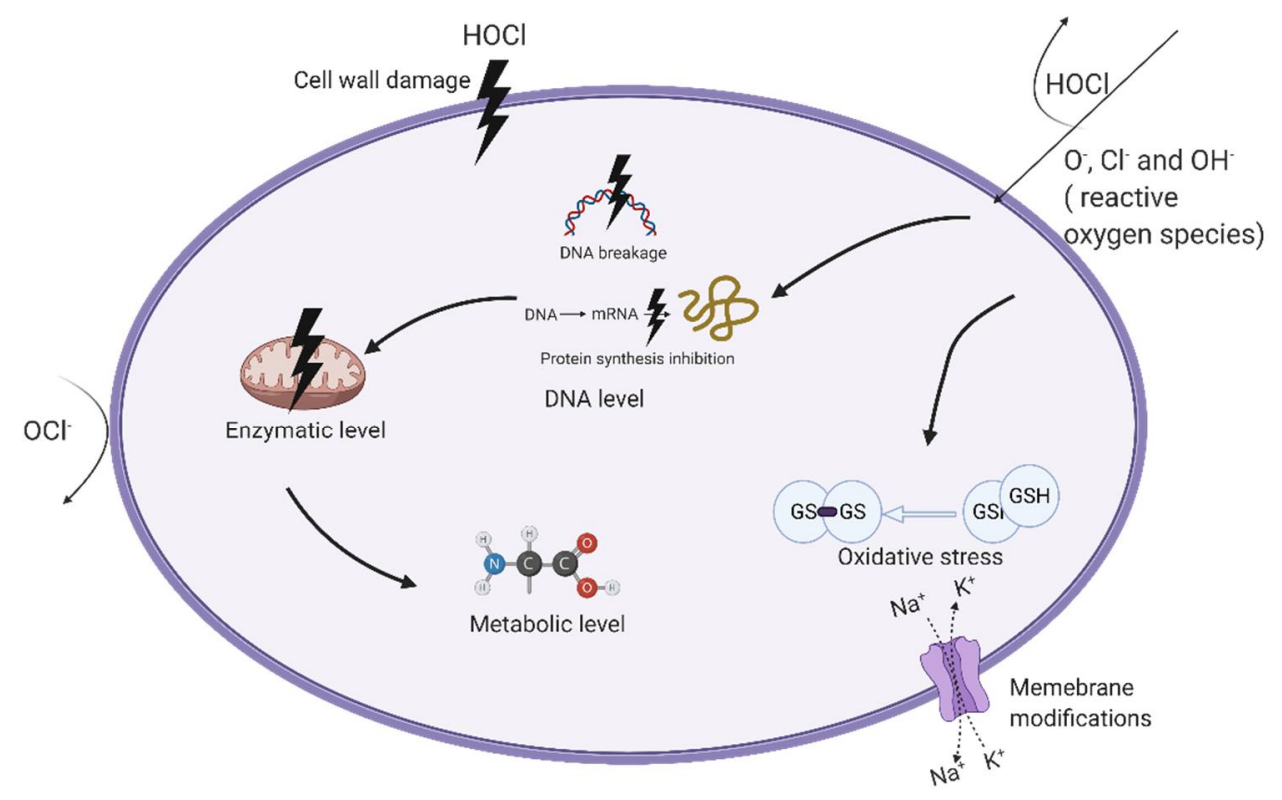

Figure 2. The germicidal mechanisms of EW on microorganisms. $\mathrm{ClO}^{-}, \mathrm{Cl}^{-}$, and $\mathrm{OH}^{-}$attack only on the cell wall and outer membrane of the cell. $\mathrm{HOCl}$ can penetrate the cell membrane's lipid bilayer. EW attacking various cellular targets including cytoderm, intracellular components, and outer membrane. $\mathrm{HOCl}$ disordered intracellular components, normal cellular functions, and bacterial ultrastructures in various degrees including complex changes of intracellular metabolites, metabolic pathway (enzymatic level), and intracellular microenvironment (membrane integrity).

In addition to the chlorine family, other compounds such as reactive oxygen species (ROS) can also be produced in electrolysis, contributing to antimicrobial efficiency [35]. After EW penetrating the microbial cell, $\mathrm{HOCl}$ produced $\mathrm{ROS}$ (such as $\mathrm{O}^{-}$and $\mathrm{OH}^{-}$) and $\mathrm{Cl}^{-}$, causing substantially disordered intracellular components, normal cellular functions, and bacterial ultrastructures in various degrees. (1) Including complex changes of intracellular metabolites, EW causes significant biochemical processes related to amino acid metabolism, peptidoglycan synthesis, nucleotides synthesis, and lipid biosynthesis $[36,37]$. Moreover, (2) in metabolic pathways (enzymatic level), EW shows negative effects on intracellular esterase activity, $\gamma$-aminobutyric acid (GABA) shunt, and enhanced glutamate decarboxylase (GAD) system for preventing oxidative stress and inactivating intracellular TTC-dehydrogenase [33,37,38]. (3) The intracellular microenvironment (membrane integrity) is also affected by breaking cell membrane integrity and decreasing membrane potentials to induce cell necrosis and apoptosis [38]. Cell membranes are the critical target for EW treatment of microorganisms [13].

In addition to lethal effects, EW could induce microorganisms to transfer into other physiological states, such as sublethal injury and viable but non-culturable (VBNC) state, in which microorganisms could wait for resuscitation when the conditions become suitable for their growth. Liu et al. reported that the sublethal injured percentage of Listeria monocytogenes and Listeria innocua was 70\% and 65\%, respectively, after treatment of EW (8 ppm) [37].

Furthermore, EW can inhibit the activities of polyphenol oxidase activity (PPO) of fresh-cut vegetables such as sweet potato slices, mushrooms, and lotus root [38-40]. PPO, 
which contributed to the brown reaction, catalyzes the oxidation of phenolic compounds into reactive quinones, and brown pigments were generated to quinones polymerization. The active compound (chlorine species: $\mathrm{HOCl}, \mathrm{Cl}_{2}$, and $\mathrm{OCl}^{-}$) of $\mathrm{EW}$ was considered to be the reason for the inhibition effect of PPO activity. EW can effectively inhibit microorganism growth and slow down the increase in TVB-N production of marine fish [41]. However, the exact pattern of EW on microbial cells is still unclear, and it requires more investigations to clarify its nature in the future.

\section{Disinfectant Based on EW in the Food Industry}

\subsection{Application of EW in Fruits and Vegetables}

Pathogens can contaminate or colonize fruits and vegetables from preharvest production, distribution, processing, and packaging to food preparation. Microbial contamination of fruits and vegetables can cause spoilage of food and even human infection and poisoning. In order to ensure the safety of fruits and vegetables for human consumption, EW has been widely proved to be one of the most advanced treatments in vegetables. The sanitizing effect of various types of EW apply for vegetables and fruits is depicted in Table 1. Shen et al. reported the effectiveness of neutral EW treatment on apples (available chlorine concentration (ACC): 100 ppm, pH 6.9, and exposure time $2 \mathrm{~min}$ ) against L. monocytogenes, Lactobacillus plantarum, Pediococcus acidilactici, and L innocua, Enterococcus faecium. A total reduction of 1.1 to $2.1 \log \mathrm{CFU} / \mathrm{g}$ was achieved for all types of bacteria in apple. Puligundla et al. also reported reductions of $1.3 \log$ for yeasts and molds and $1.4 \log$ and $1.0 \log \mathrm{CFU} / \mathrm{g}$ for total aerobic bacteria when broccoli sprout was dipped in AEW for $20 \mathrm{~s}$ [42].

Table 1. Application of EW in disinfecting various vegetables and fruits.

\begin{tabular}{|c|c|c|c|c|c|c|c|c|}
\hline EW Type & $\begin{array}{c}\text { Food } \\
\text { Commodities }\end{array}$ & Microorganisms & $\begin{array}{c}\text { Log } \\
\text { Reduction } \\
\text { (log CFU/g) }\end{array}$ & $\begin{array}{l}\text { Exposure } \\
\text { Time } \\
\text { (min) }\end{array}$ & $\begin{array}{l}\text { Free Chlorine } \\
\text { Concentration } \\
\text { (ppm) }\end{array}$ & $\mathrm{pH}$ & ORP & Reference \\
\hline SAEW & Pea sprout & $\begin{array}{c}\text { Total bacteria count } \\
\text { Coliform } \\
\text { Yeast and mold }\end{array}$ & $\begin{array}{l}1.58 \\
1.02 \\
1.22\end{array}$ & $24 \mathrm{~h}$ & 70 & 5.46 & 927 & [23] \\
\hline SAEW & $\begin{array}{c}\text { Spinach } \\
\text { Beet } \\
\text { Lettuce leaves }\end{array}$ & B. cereus & $\begin{array}{l}2.7 \\
3.0 \\
2.91\end{array}$ & 15 & 80 & 5.74 & $832-855$ & [43] \\
\hline SAEW & $\begin{array}{l}\text { Buckwheat } \\
\text { sprouts }\end{array}$ & $\begin{array}{c}\text { Escherichia coli O78 } \\
\text { L.monocytogenes }\end{array}$ & $\begin{array}{l}2.74 \\
2.46\end{array}$ & 5 & 91.96 & 6.26 & 831 & {$[44]$} \\
\hline EW & Lettuce & Salmonella spp. & 4 & $45 \mathrm{~s}$ & 50 & - & - & [45] \\
\hline NEW & Apples & L. monocytogenes & 1.0 & 2 & 110 & 6.88 & 883.7 & [46] \\
\hline EOW & Lettuce & $\begin{array}{c}\text { E. coli } \\
\text { Salmonella enteritidis } \\
\text { L. innocua } \\
\text { E.coli } \\
\text { S. Enteritidis }\end{array}$ & $\begin{array}{l}1.2 \\
1.0 \\
1.3 \\
1.5 \\
1.8\end{array}$ & 10 & 50 & 6.8 & 855 & [47] \\
\hline NEW & Spinach leaves & E. coli & 0.3 & 1 & 85 & 7.0 & 857 & [48] \\
\hline SAEW & $\begin{array}{l}\text { Wheat grains } \\
\text { straight flour } \\
\text { clear flour } \\
\text { bran }\end{array}$ & Total plate count & $\begin{array}{l}0.65 \\
0.72 \\
0.91 \\
0.93\end{array}$ & 1.5 & 70 & - & - & [49] \\
\hline NEW & Strawberries & MS2 bacteriophage & 0.9 & 1.5 & $50-55$ & - & - & [50] \\
\hline NEW & Apple & $\begin{array}{l}\text { L. monocytogenes } \\
\text { L. plantarum } \\
\text { P. acidilactici } \\
\text { L. innocua } \\
\text { E. faecium }\end{array}$ & $\begin{array}{l}1.1 \\
2.1 \\
1.3 \\
1.2 \\
1.1\end{array}$ & 2 & 110 & 6.9 & 883.7 & [51] \\
\hline
\end{tabular}


Table 1. Cont.

\begin{tabular}{|c|c|c|c|c|c|c|c|c|}
\hline EW Type & $\begin{array}{c}\text { Food } \\
\text { Commodities }\end{array}$ & Microorganisms & $\begin{array}{c}\text { Log } \\
\text { Reduction } \\
\text { (log CFU/g) }\end{array}$ & $\begin{array}{l}\text { Exposure } \\
\text { Time } \\
\text { (min) }\end{array}$ & $\begin{array}{l}\text { Free Chlorine } \\
\text { Concentration } \\
\quad(\mathrm{ppm})\end{array}$ & $\mathrm{pH}$ & ORP & Reference \\
\hline SAEW & $\begin{array}{l}\text { Celery } \\
\text { Cilantro }\end{array}$ & $\begin{array}{l}\text { Total aerobic bacteria } \\
\text { Yeast and mold } \\
\text { Total aerobic bacteria } \\
\text { Yeast and mold }\end{array}$ & $\begin{array}{l}4.63 \\
4.17 \\
4.66 \\
3.86\end{array}$ & 7 & 25 & - & - & [52] \\
\hline SAEW & Cut cabbage & $\begin{array}{l}\text { Pectobacterium } \\
\text { carotovorum subsp. } \\
\text { carotovorum }\end{array}$ & 5.94 & $180 \mathrm{~s}$ & 22.17 & 5.0 & 950-980 & [53] \\
\hline
\end{tabular}

Notes: ORP: Oxidation-reduction potential; EOW: electrolyzed oxidizing water. SAEW: slightly acidic electrolyzed water; AEW: acidic electrolyzed water; NEW: neutralized electrolyzed water; EW: electrolyzed water. ACC: available chlorine concentration.

Furthermore, Tang et al. reported that AEW treatments (ACC: $80 \mathrm{mg} / \mathrm{L}, \mathrm{pH} 2.5$, exposure time: $10 \mathrm{~min}$ ) reduced disease occurrence in harvested longans. AEW treatment maintained high levels of non-enzymatic antioxidant systems and increased the activities of antioxidant enzymes to maintain longer shelf life [54]. The disinfection efficacy of SAEW treatment on wheat grains and pea sprout was also studied, and the total number of bacteria was reduced by approximately 0.65 to 1.58 log reductions, respectively $[23,49]$. Ogunniyi et al. reported that NEW was applied as a viable alternative to chemically based sanitizers for post-harvest treatment of spinach leaves [48].

The key factors are exposure time and temperature to reduce microbial count in fresh vegetables and fruits. Cap et al. evaluated the effectiveness of EW against Salmonella spp. in lettuce after short exposure times of $15 \mathrm{~s}, 30 \mathrm{~s}$, and $45 \mathrm{~s}$. The results revealed a Salmonella $\log$ reduction of $2.90 \log \mathrm{CFU} / \mathrm{g}$ after $15 \mathrm{~s}$ and $30 \mathrm{~s}$ and $4.06 \mathrm{log} \mathrm{CFU} / \mathrm{g}$ for treatment for $45 \mathrm{~s}$ [45]. However, Stopforth et al. studied AEW (ACC 50 ppm) in leafy greens and revealed no significant difference in the antibacterial effect above $1 \mathrm{~min}$. Some other authors studied the effectiveness of EW for longer exposure times and reported similar or even lower bacterial reductions $[55,56]$. The reason may be that the dissociation of free chlorine or interference such as organic matter over a relatively short time [57]. Some other scientists reported that the storage temperature of fresh-cut vegetables should be below $5{ }^{\circ} \mathrm{C}$ in order to reduce the proliferation of spoilage and pathogenic microorganisms [58].

\subsection{Application of EW in Poultry and Meat}

Microbial contamination of poultry and meat products is a vital factor contributing to quality losses and foodborne illnesses. Many novel and effective chemical technologies, including EW treatment, have been used to reduce microbial contamination in meat and poultry. Around the world, $36 \%$ of all the meat consumed is pork, followed by poultry meat and beef at $35 \%$ and $22 \%$, respectively [59].

EW's effectiveness has been reported against populations of S. enterica, E. coli, Yersinia inoculated, and Staphylococcus aureus, which are often associated with pork, beef, chicken, and other meat surfaces [60]. The decontamination efficiency of EW on poultry and meat products is depicted in Table 2. Recently, most studies have used soaking, spray, or immersion techniques with varying degrees of success [60-62]. Murad et al. achieved 1.5-1.6 logs CFU/g reduction in E. coli O157:H7 and Salmonella typhymurium on inoculated chickens and beef after $10 \mathrm{~min}$ of soaking in AEW treatment [63]. Wang et al. discovered that almost $1.0 \log \mathrm{CFU} / \mathrm{cm}^{2}$ microbial reduction was achieved by using novel spraying technology [62]. Han et al. studied the effectiveness of NEW on pork skin inoculated with E. coli O157:H7, Yersinia enterocolitica, and Salmonella. After 2 min of immersion treatment with NEW containing $74 \mathrm{ppm}$, total reductions of 2.12, 2.22, and $1.74 \log \mathrm{CFU} / \mathrm{g}$ were recorded for E. coli O157: H7, S. enteritidis, and Y. enterocolitica [64]. SAEW with neutral $\mathrm{pH}$ value was effective for reducing the population of total aerobic bacteria (TAB), total viable counts (TVC), total coliforms mesophilic count, and even yeast and mold, while maintaining the properties of pork, chicken, and beef including increased shelf life, enhanced acceptable sensory properties, and delayed lipid oxidation [63,65]. 
Table 2. Application of EW against various microorganism in poultry and meat.

\begin{tabular}{|c|c|c|c|c|c|c|c|c|}
\hline EW Type & $\begin{array}{c}\text { Food } \\
\text { Commodities }\end{array}$ & Microorganisms & $\begin{array}{c}\text { Log } \\
\text { Reduction } \\
\text { (log CFU/g) }\end{array}$ & $\begin{array}{l}\text { Exposure Time } \\
\text { (min) }\end{array}$ & $\begin{array}{c}\mathrm{ACC} \\
(\mathrm{ppm})\end{array}$ & $\mathrm{pH}$ & ORP & Reference \\
\hline SAEW & $\begin{array}{c}\text { Beef } \\
\text { Chicken } \\
\text { Pork }\end{array}$ & Total viable count & $\begin{array}{l}2.7 \\
3.1 \\
2.7\end{array}$ & 10 & $29-30$ & $856-875$ & $5.47-5.50$ & [28] \\
\hline NEW & Pork skin & $\begin{array}{l}\text { E. coli O157:H7 } \\
\text { S. enteritidis } \\
\text { Yersinia-inoculated }\end{array}$ & $\begin{array}{l}2.12 \\
2.59 \\
2.22 \\
2.37 \\
1.74 \\
1.81\end{array}$ & $\begin{array}{c}2 \\
10 \\
2 \\
10 \\
2 \\
10\end{array}$ & 74 & 7.64 & 818 & [64] \\
\hline NEW & Eggshells & $\begin{array}{l}\text { S. enterica } \\
\text { E. coli }\end{array}$ & $\begin{array}{l}99.99 \% \\
97.34 \% \\
\end{array}$ & 0.5 & 60.85 & 7.12 & 907.27 & [66] \\
\hline SAEW & Beef & Total viable count & 1.43 & 3 & 30 & 6.29 & $870-900$ & [67] \\
\hline SAEW & Slicing Blades & L. monocytogenes & $\begin{array}{l}1.4 \\
3.6\end{array}$ & 30 s spray & $\begin{array}{c}5 \\
25 \\
\end{array}$ & $6.4-6.6$ & - & [68] \\
\hline NEW & Chicken meat & Total viable count & 1.2 & $90 \mathrm{~min}$ & 50 & - & - & [69] \\
\hline LCAEW & Pork & $\begin{array}{l}\text { Total number of } \\
\text { microorganisms } \\
\text { Yeast and moulds } \\
\text { Psychrotrophs }\end{array}$ & $\begin{array}{c}3.25 \\
2.68 / \mathrm{cm}^{2} \\
3.10\end{array}$ & spray & 16.6 & 2.15 & 1159.32 & [70] \\
\hline AEW & $\begin{array}{c}\text { Beef } \\
\text { Chevon } \\
\text { Pork }\end{array}$ & E. coli $\mathrm{K} 12$ & $\begin{array}{l}1.16 \\
1.22 \\
1.3\end{array}$ & $\begin{array}{c}4 \\
12 \\
10\end{array}$ & 34.3 & 759.9 & 3.03 & [71] \\
\hline SAEW & Beef steaks & Total viable count & 2.35 & 1.5 & 30 & 8 & 5.85 & [72] \\
\hline NEW & Pork chops & L. monocytogenes & $99.98 \%$ & 1.0 & 58 & 820 & 6.92 & [73] \\
\hline
\end{tabular}

Notes: ORP: Oxidation-reduction potential; NEW: neutralized electrolyzed water; SAEW: slightly acidic electrolyzed water; AEW: acidic electrolyzed water. ACC: available chlorine concentration.

\subsection{Application of EW in Aquatic Product}

Aquatic products contain high quality proteins, which are easily polluted by spoilage bacteria or pathogenic from the process of production, transportation to sale [74]. These bacteria not only reduce seafood quality and shorten shelf life but also pose a potential risk to human health. Currently, EW technology has been used for safety control and preservation of aquatic products, and these are shown in the Table 3. Hamzah et al. demonstrated that AEW ice treatment exhibited visible bacterial reduction on mussel and clam for $1 \mathrm{~h}$ [75]. Xuan et al. studied that SAEW ice could control microbial safety and quality deterioration of squid [76]. SAEW ice treatment could delay browning and softening appearances, maintain lower thiobarbituric acid (TBA) and total volatile basic nitrogen (TVEN) content, and inhibit the incensement of peroxide value. Furthermore, Zhao et al. investigated the changes of key proteins on shrimps during AEW ice storage [77]. The results showed that AEW ice significantly retarded the degradation of myofibrillar proteins in shrimps, including actin, myosin, and tropomyosin. The combination of EW with other technology in aquatic products is discussed in the hurdle technology section in more detail. 
Table 3. Application of EW against various microorganism in aquatic products.

\begin{tabular}{|c|c|c|c|c|c|c|c|c|}
\hline EW Type & Food Commodities & Microorganisms & $\begin{array}{c}\text { Log } \\
\text { Reduction } \\
\text { (log CFU/g) }\end{array}$ & $\begin{array}{l}\text { Exposure } \\
\text { Time } \\
\text { (min) }\end{array}$ & $\begin{array}{c}\mathrm{ACC} \\
(\mathrm{ppm})\end{array}$ & $\mathrm{pH}$ & ORP & Reference \\
\hline \multirow{4}{*}{ AEW } & \multirow[t]{2}{*}{$\underset{\text { (Venerupis philippinarum) }}{\text { Clam }}$} & $\begin{array}{c}\text { Escherichia coli } \\
\text { O104:H4 }\end{array}$ & 1.4 & \multirow{4}{*}{$1 \mathrm{~h}$} & \multirow{4}{*}{20} & \multirow{4}{*}{3.1} & \multirow{4}{*}{1150} & \multirow{4}{*}[75]{} \\
\hline & & $\begin{array}{l}\text { Listeria monocytogenes } \\
\text { Aeromonas hydrophila }\end{array}$ & $\begin{array}{l}1.0 \\
1.4\end{array}$ & & & & & \\
\hline & \multirow{2}{*}{$\begin{array}{c}\text { Mussel } \\
\text { (Mytilus edulis) }\end{array}$} & $\begin{array}{c}\text { Vibrio } \\
\text { parahaemolyticus }\end{array}$ & 1.3 & & & & & \\
\hline & & Campylobacter jejuni & 1.5 & & & & & \\
\hline \multirow{4}{*}{ AEW } & \multirow{2}{*}{$\begin{array}{c}\text { Clam } \\
\text { (Venerupis philippinarum) }\end{array}$} & $\begin{array}{c}\text { Escherichia coli } \\
\text { O104:H4 }\end{array}$ & 1.6 & \multirow{4}{*}{$1 \mathrm{~h}$} & \multirow{4}{*}{20} & \multirow{4}{*}{3.1} & \multirow{4}{*}{1150} & \multirow{4}{*}[75]{} \\
\hline & & $\begin{array}{l}\text { Listeria monocytogenes } \\
\text { Aeromonas hydrophila }\end{array}$ & $\begin{array}{l}1.3 \\
1.3\end{array}$ & & & & & \\
\hline & \multirow{2}{*}{$\begin{array}{c}\text { Mussel } \\
\text { (Mytilus edulis) }\end{array}$} & Vibrio & 1.0 & & & & & \\
\hline & & $\begin{array}{c}\text { parahaemolyticus } \\
\text { Campylobacter jejuni }\end{array}$ & 2.1 & & & & & \\
\hline $\begin{array}{l}\text { SAEW } \\
\text { ice }\end{array}$ & Squid & TVC & 1.48 & 1 day & 25 & 6.48 & 882 & [76] \\
\hline $\begin{array}{l}\text { SAEW } \\
\text { ice }\end{array}$ & Brown sole & $\begin{array}{l}\text { Total bacterial count } \\
\text { Pseudomonas spp. }\end{array}$ & $\begin{array}{l}\text { Extend the } \\
\text { shelf life }\end{array}$ & 11 days & 45 & 5.07 & - & [78] \\
\hline SAEW & Prawn & Total viable counts & 1.09 & $5 \mathrm{~min}$ & 20 & 5.92 & 810 & [79] \\
\hline SAEW & Oyster & $\begin{array}{c}\text { TVC } \\
\text { S. aureus } \\
\text { V. parahaemolyticus }\end{array}$ & $\begin{array}{l}1.35 \\
1.01 \\
3.00\end{array}$ & $30 \mathrm{~min}$ & 60 & 6.14 & - & [80] \\
\hline
\end{tabular}

Notes: ORP: Oxidation-reduction potential; NEW: neutralized electrolyzed water; SAEW: slightly acidic electrolyzed water; AEW: acidic electrolyzed water. ACC: available chlorine concentration. TVC: Total viable counts.

\subsection{Hurdle Enhancement of EW with Other Treatments}

EW is an effective broad-spectrum antimicrobial agent with several advantages: on the spot, cheap, environmentally friendly, and safety production [81]. However, it is necessary to face and solve the problem that EW is still unstable and insufficient for completely inactivating or decontaminating many food or other products [82]. In addition, the demand for organoleptic quality and fresh and nutritious food requires the development of novel technologies for prolonging shelf life. Currently, hurdle technologies combing two or more preservative and sanitizing technologies can improve inactivation efficacy without undesirable effects on food quality [83]. EW combined with other technologies has been widely studied for more than 15 years. Hurdle enhancement of EW has attracted much attention due to its synergistic microbial effectiveness on targeted microorganisms. Many studies have evaluated a significant reduction in microbial loads in various food products after treatment with a combination of EW and chemical treatments. EW, as a combination, has been used in different ways, including simultaneous hurdles and sequential hurdles, and is shown in Table 4. 
Table 4. Combination of EW with other treatment procedures to decontaminate food.

\begin{tabular}{|c|c|c|c|c|c|c|c|}
\hline Combination Type & ACC & $\mathrm{pH}$ & $\mathrm{mV}$ & Food Product & Microorganisms & $\begin{array}{l}\text { Reduction } \\
\text { (log CFU/g) }\end{array}$ & Reference \\
\hline $\begin{array}{l}\text { LCEW }(3 \mathrm{~min})+\mathrm{US} \\
(3 \mathrm{~min})\end{array}$ & 16.85 & 5.5 & 513.6 & Mushroom & $\begin{array}{l}\text { Total bacterium counts } \\
\text { Yeasts and molds }\end{array}$ & $\begin{array}{c}1.1 \\
1.87\end{array}$ & [39] \\
\hline $\mathrm{SAEW}+\mathrm{US}$ at $60^{\circ} \mathrm{C}$ & 80 & 5.74 & $832-855$ & $\begin{array}{l}\text { Spinach } \\
\text { Beet leaf }\end{array}$ & 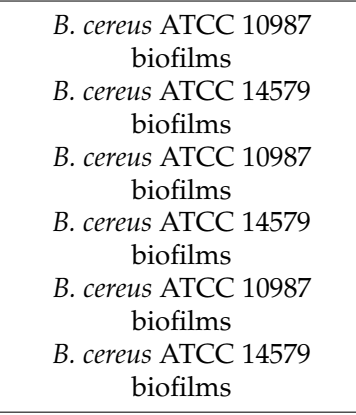 & $\begin{array}{c}2.7 \mathrm{log} / \mathrm{cm}^{2} \\
3.45 \mathrm{log} / \mathrm{cm}^{2} \\
3.0 \mathrm{log} / \mathrm{cm}^{2} \\
3.38 \mathrm{log} / \mathrm{cm}^{2} \\
2.91 \mathrm{log} / \mathrm{cm}^{2} \\
3.41 \mathrm{log} / \mathrm{cm}^{2}\end{array}$ & [43] \\
\hline Spray + SAEW & 30 & 6.0 & 845 & Chicken & Total bacterial count & $1.0 \log \mathrm{CFU} / \mathrm{cm}^{2}$ & [62] \\
\hline Ozone + EW & 10 & 3 & 663 & $\begin{array}{l}\text { Alfalfa seeds } \\
\text { Alfalfa sprouts }\end{array}$ & $\begin{array}{c}\text { S. enterica } \\
\text { Shiga toxin-producing } \\
\text { E. coli } \\
\text { S. enterica } \\
\text { Shiga toxin-producing } \\
\text { E. coli }\end{array}$ & $\begin{array}{l}3.6 \\
2.9 \\
\\
3.1 \\
3.0\end{array}$ & [84] \\
\hline $\begin{array}{l}\text { AEW + levulinic acid } \\
(3 \% v / v) \text { for } 7 \mathrm{~min}\end{array}$ & 4 & 3.84 & 920.5 & Lettuces & $\begin{array}{c}\text { E. coli ATCC } 25922 \\
\text { L. innocua Seeliger ATCC } \\
33090 \\
\text { Aerobic mesophilic counts } \\
\text { Yeasts and moulds }\end{array}$ & $\begin{array}{c}3.5-4 \\
3.5-4 \\
4.0 \\
4.5\end{array}$ & [85] \\
\hline $\begin{array}{c}\text { SAEW }+0.5 \% \\
\text { fumaric acid at } 40{ }^{\circ} \mathrm{C} \\
\text { for } 3 \mathrm{~min}\end{array}$ & 15 & 2.95 & 1091 & Pork & $\begin{array}{l}\text { E.coli O157:H7 } \\
\text { L. monocytogenes } \\
\text { S. aureus } \\
\text { S. Typhimurium }\end{array}$ & $\begin{array}{l}2.59 \\
2.69 \\
2.38 \\
2.99\end{array}$ & [86] \\
\hline $\begin{array}{l}\text { SAEW + BAC + } \\
60^{\circ} \mathrm{C} \text { for } 10 \mathrm{~min}\end{array}$ & 40 & - & - & Pure culture & $\begin{array}{l}\text { Bacillus cereus ATCC } 10987 \\
\text { B. cereus ATCC } 14579 \\
\text { ATCC } 10987 \text { biofilms }\end{array}$ & $\begin{array}{l}1.91 \log \mathrm{CFU} / \mathrm{mL} \\
1.98 \log \mathrm{CFU} / \mathrm{mL} \\
2.62 \log \mathrm{CFU} / \mathrm{cm}^{2}\end{array}$ & [87] \\
\hline $\begin{array}{l}\text { SAEW }(5 \mathrm{~min})+ \\
\text { US }(100 \mathrm{~min})\end{array}$ & 30 & 5.8 & 810 & Sliced shad & $\begin{array}{c}\text { E. coli } 10536 \\
\text { V. parahaemolyticus KCTC } \\
2471\end{array}$ & $\begin{array}{l}1.86 \\
1.42\end{array}$ & [88] \\
\hline $\begin{array}{l}\text { Heat treatment } \\
\left(55^{\circ} \mathrm{C} \text { for } 10 \mathrm{~min}\right)+ \\
\text { SAEW }(30 \mathrm{~s})\end{array}$ & 30 & 6.3 & 867.4 & Pure culture & L. monocytogenes & $2.91 \log \mathrm{CFU} / \mathrm{mL}$ & [89] \\
\hline $\begin{array}{l}\text { LCEW + 3\% calcium } \\
\text { lactate for } 5 \text { min }\end{array}$ & 10 & 6.8 & 700 & Pork & $\begin{array}{l}\text { Microbial load } \\
\text { Yeasts and molds } \\
\text { L. monocytogenes } \\
\text { E. coli O157:H7 }\end{array}$ & $\begin{array}{c}2.20 \\
1.57 \\
3.1 \\
2.97\end{array}$ & [90] \\
\hline SAEW + US (25 khz) & 25 & 7.0 & $130-140$ & Chicken breast & $\begin{array}{l}\text { Psychrotrophic bacteria } \\
\text { Mesophilic bacteria }\end{array}$ & $\begin{array}{l}0.76 \\
0.98\end{array}$ & [91] \\
\hline $\begin{array}{l}\text { AkEW }+1 \% \text { citric } \\
\text { acid at } 50{ }^{\circ} \mathrm{C} \text { for } \\
10 \mathrm{~min}\end{array}$ & 50 & $11-11.2$ & $\begin{array}{l}-830 \text { to } \\
-850\end{array}$ & Cabbage & $\begin{array}{l}\text { Total count } \\
\text { Yeast and mold } \\
\text { L. Monocytogenes } \\
\text { E. coli O157:H7 }\end{array}$ & $\begin{array}{l}3.98 \\
3.45 \\
3.99 \\
4.19\end{array}$ & [92] \\
\hline $\begin{array}{c}\text { SAEW + } \\
\text { thermosonication }\end{array}$ & 5 & 6.3 & 890 & Kale & $\begin{array}{c}\text { Aerobic mesophilic bacteria } \\
\text { Pseudomonas spp. }\end{array}$ & $\begin{array}{l}2.84 \\
2.52 \\
\end{array}$ & [93] \\
\hline AEW + ultrasonic & 31.7 & 3.2 & 943 & Pure culture & B.cereus ATCC 14579 & $2.29 \log \mathrm{CFU} / \mathrm{mL}$ & [94] \\
\hline $\begin{array}{l}\text { SAEW }(5 \mathrm{~min})+ \\
\text { UV-LED }(30 \mathrm{~min})\end{array}$ & 60 & - & - & $\begin{array}{l}\text { Coriander } \\
\text { leaves }\end{array}$ & $\begin{array}{c}\text { Salmonella Typhimurium } \\
\text { strains } \\
\text { E. coli O157:H7 strains }\end{array}$ & $\begin{array}{l}2.72 \\
2.42\end{array}$ & [95] \\
\hline $\begin{array}{c}\text { AEW }(10 \text { min })+ \\
\text { US }\end{array}$ & 2 & 4.95 & 746 & Pure culture & B. cereus ATCC 14579 & $1.98 \log \mathrm{CFU} / \mathrm{mL}$ & [96] \\
\hline AEW + US & 30 & 2.66 & 1076 & Pure culture & Salmonella spp. biofilm & $4.8 \log \mathrm{CFU} / \mathrm{cm}^{2}$ & [97] \\
\hline
\end{tabular}


Table 4. Cont.

\begin{tabular}{|c|c|c|c|c|c|c|c|}
\hline Combination Type & ACC & $\mathrm{pH}$ & $\mathbf{m V}$ & Food Product & Microorganisms & $\begin{array}{l}\text { Reduction } \\
\text { (log CFU/g) }\end{array}$ & Reference \\
\hline $\mathrm{EW}+$ heat treatment & 4 & 4.02 & 956.8 & Organic carrot & E. coli O157:H7 & 3.5 & [98] \\
\hline \multirow{2}{*}{$\begin{array}{l}\mathrm{NEW}+\text { heating } \\
\left(65^{\circ} \mathrm{C}\right) \text { for } 10 \mathrm{~min} \\
\mathrm{AEW}+\text { heating } \\
\left(65^{\circ} \mathrm{C}\right) \text { for } 10 \mathrm{~min}\end{array}$} & 60 & 6.8 & 786 & $\begin{array}{c}\text { Atlantic } \\
\text { salmon fillets }\end{array}$ & L. monocytogenes & 5.0 & \multirow[t]{2}{*}{ [99] } \\
\hline & 60 & 2.7 & 1150 & $\begin{array}{c}\text { Atlantic } \\
\text { salmon fillets }\end{array}$ & L. monocytogenes & 5.6 & \\
\hline AEW-HHP (400mpa) & $36-73$ & $\begin{array}{l}2.28- \\
2.39\end{array}$ & 1077-1168 & Shrimp & $\begin{array}{l}\text { V. parahaemolyticus } \\
\text { L. monocytogenes }\end{array}$ & $\begin{array}{l}6.08 \\
5.71\end{array}$ & [100] \\
\hline $\begin{array}{c}\text { SAEW + } \\
\text { Bamoon extracts }\end{array}$ & 27.37 & 5.5 & 836 & Bombay duck & Total viable count & $\begin{array}{c}\text { Prolong } 12 \text { days of } \\
\text { shelf life compared } \\
\text { to untreatment }\end{array}$ & [101] \\
\hline \multirow{2}{*}{$\begin{array}{c}\text { BEW }(20 s)+A E W \\
(20 s)\end{array}$} & \multirow{2}{*}{23} & \multirow{2}{*}{2.6} & \multirow{2}{*}{1185} & \multirow{2}{*}{ Pork loin } & \multirow{2}{*}{$\begin{array}{c}\text { Mesophilic microorganisms } \\
\text { Psychrotrophs } \\
\text { microorganisms }\end{array}$} & 0.46 & \multirow{2}{*}{ [102] } \\
\hline & & & & & & 0.82 & \\
\hline \multirow{3}{*}{$\begin{array}{c}\text { UV }(5 \min )+\text { US } \\
(1 \mathrm{~min})+\text { AEW } \\
(1 \mathrm{~min})\end{array}$} & \multirow{3}{*}{65} & \multirow{3}{*}{2.6} & \multirow{3}{*}{1140} & \multirow{3}{*}{ Salmon fillets } & L. monocytogenes & 0.75 & \multirow{3}{*}{ [103] } \\
\hline & & & & & & & \\
\hline & & & & & Total viable count & 0.64 & \\
\hline \multirow{3}{*}{$\begin{array}{l}\text { AEW (washing) + } \\
0.5 \% \text { lysozyme } \\
\text { enzyme solution }\end{array}$} & \multirow{3}{*}{100} & \multirow{3}{*}{2.5} & \multirow{3}{*}{-} & \multirow{3}{*}{$\begin{array}{l}\text { Scaly } \\
\text { common } \\
\text { carps }\end{array}$} & Total viable count & 2.88 & \multirow{3}{*}{ [104] } \\
\hline & & & & & Enterobacteriaceae count & 1.43 & \\
\hline & & & & & Anaerobic mesophilic count & 2.98 & \\
\hline
\end{tabular}

Notes: AEW: acidic electrolyzed water; SAEW: slightly acidic electrolyzed water; LCEW: low concentration electrolyzed water; AkEW: alkaline electrolyzed Water. ACC: available chlorine concentration.

However, a practical synergistic effect was observed when EW is combined with organic acids (citric acid [CA], fumaric acid [FA], and lactic acid), essential oils, benzalkonium chloride, etc., when simultaneously used against foodborne pathogens on food products. Rahman et al. investigated the combined sanitizing effects of AkEW with $1 \% \mathrm{CA}$ at $50{ }^{\circ} \mathrm{C}$ on fresh cabbage [92]. Their results revealed a significantly reduced effective reduction in E. coli O157:H7 (4.19 log CFU/g) and L. monocytogenes (3.99 log CFU/g). Tango et al. found that $\mathrm{CaO}$ washing tomato and apple followed by SAEW + FA showed effective decontamination. Moreover, the softened skin of tomato fruits can be maintained by this method due to the inclusion of calcium [105]. In addition to EW, organic acids also significantly influence disinfection power. It is well known that organic acids have great potential for controlling a wide range of microorganisms. The main reason for the antimicrobial effect is the lipophilic nature of the undissociated form of organic acids and proton modification.

Ahmad et al. also studied that SAEW combined with $0.5 \%$ FA on pork showed a more significant bactericidal effect and prolonged shelf life at 6 days $[106,107]$. Consequently, bacterial viability decreased because essential enzymes and purine bases are negatively affected [108]. Hence, the synergistic effect of EW and organic acid could eliminate or inhibit brand microbes, organoleptic quality enhancement, and shelf-life prolongation by using different mechanisms [86,109].

Treatment with the combination of EW and physical sanitizing techniques has also been studied to guarantee microbiological safety and quality of the final product. For example, ultraviolet irradiation, ultrasound, heat treatment, sonication, and multi pulsed, high pressure have been successfully applied to subsequent treatment with EW against foodborne pathogens. Wu et al. reported that low concentrations of AEW combined with ultrasound (US) had a better bactericidal effect on mushrooms than individual methods [39]. Recently, Zahra et al. studied the effect of AEW/ozone combination against Salmonella on the quality of alfalfa seeds and sprouts. Their results showed a 3.6 and $2.9 \log$ CFU/g reduction of seeds and sprouts, respectively, while no significant changes in the quality were observed, including shelf life, weight, and color.

Currently, Jiang et al. also studied the effect of the ultraviolet light-emitting diode in combination with SAEW against Salmonella and E. coli O157:H7 on the surfaces of coriander. The results revealed that the above method resulted in a $2.72 \log \mathrm{CFU} / \mathrm{g}$ 
Salmonella reduction and $2.42 \log \mathrm{CFU} / \mathrm{g}$ E. coli reduction and did not adversely affect the quality and increased shelf life [95]. The treatment SAEW combined with ultraviolet (UV) radiation also improves internal egg quality during six weeks of storage [110].

Furthermore, the use of EW combined with chemical and physical treatments to improve antimicrobial activity has been studied by several researchers. When the combination of benzalkonium chloride (BAC) and SAEW at $60^{\circ} \mathrm{C}$ was investigated on vegetative cells, spores, and biofilms, a synergistic antimicrobial effect was observed, which resulted in reductions of ATCC 10,987 biofilms, ATCC 14,579 spores, and ATCC 10,987 spores at $2.62 \log \mathrm{CFU} / \mathrm{cm}^{2}, 1.98 \log \mathrm{CFU} / \mathrm{mL}$, and $1.91 \log \mathrm{CFU} / \mathrm{mL}$, respectively [87]. EW-based hurdles overcome certain limitations of individual treatment and maintained maximum food quality and safety attributes. These novel hurdle inactivation approaches could be an effective strategy against pathogens in food products, food contact surfaces, and medical instruments.

\subsection{Clinical Application of EW in Hospitals}

The demand for sanitizers and disinfectants in the health care and medical sectors has increased worldwide since the outbreak of COVID-19. The U.S. Environmental Protection Agency has recommended many disinfectants against COVID-19, including HOCl. In addition, owing to EW with a $\mathrm{pH}$-neutral, safe, and broad-spectrum bactericide, the Ministry of Health of the People's Republic of China released three Chinese standards for materials and restricted substances in disinfectants, general requirements for hand disinfectants in April 2020, and general requirements for the disinfectants of mucous membrane [14]. EW can be used to disinfect medical instruments, clinical environments, and object surfaces and it can be used for disinfecting hands, skin, and mucous membranes, as shown in the Table 5 .

Table 5. Applications of EW against various microorganisms in clinical infections.

\begin{tabular}{|c|c|c|c|c|c|c|c|c|}
\hline Application & Target & $\begin{array}{l}\text { EW Type } \\
\text { (Product) }\end{array}$ & $\begin{array}{l}\text { Exposure } \\
\text { Time }\end{array}$ & $\begin{array}{l}\text { Observation } \\
\text { (log CFU) }\end{array}$ & $\begin{array}{c}\mathrm{ACC} \\
(\mathrm{ppm})\end{array}$ & $\mathrm{pH}$ & $\begin{array}{l}\text { ORP } \\
(\mathrm{Mv})\end{array}$ & Reference \\
\hline $\begin{array}{l}\text { Upper } \\
\text { respiratory } \\
\text { tract } \\
\text { hygiene }\end{array}$ & SARS-CoV-2 & AEW & $1 \mathrm{~min}$ & $\begin{array}{c}>99.8 \% \text { virucidal } \\
\text { efficacy } \\
\text { non-irritant on oral }\end{array}$ & $95 \%$ & $2.5-3$ & 1000-1200 & [111] \\
\hline $\begin{array}{l}\text { Chronic } \\
\text { wounds }\end{array}$ & $\begin{array}{l}\text { Candida albicans } \\
\text { biofilms }\end{array}$ & $\begin{array}{c}\mathrm{HOCl}- \\
\text { generating } \\
\text { electrochemical }\end{array}$ & $24 \mathrm{~h}$ & $\begin{array}{c}5 \cdot 28 \log 10 \\
\left(\mathrm{CFU} \mathrm{cm} \mathrm{cm}^{-2}\right)\end{array}$ & - & - & - & [112] \\
\hline $\begin{array}{c}\text { Atopic } \\
\text { dermatitis }\end{array}$ & $\begin{array}{l}\text { NC/Nga mouse } \\
\text { model of Atopic } \\
\text { dermatitis }\end{array}$ & EW & Twice a day & $\begin{array}{c}\text { Nontoxicity } \\
\text { less skin lesions } \\
\text { prevent scratching } \\
\text { bouts }\end{array}$ & 500 & 6.0 & - & [113] \\
\hline Mouthwash & S. mutans & EW & $1 \mathrm{~min}$ & $\begin{array}{l}>99.9 \% \\
\text { little in vivo toxicity }\end{array}$ & 12.5 & - & - & [114] \\
\hline
\end{tabular}

Notes: ACC: available chlorine concentration.

$\mathrm{HOCl}$ is the approved core formula, which has been incorporated into topical formulations due to its antimicrobial, immunomodulatory, anti-inflammatory, and wound healing properties. Currently, there are a variety of disinfection products based on EW in the market. In addition, its $\mathrm{pH}$ neutralization can enhance skin tolerability, therapeutic activity, and stability [115]. Many patents, including EW application for dental care, eye care, advanced tissue care, hand sanitizer, and dermatology, are available. EW with an antimicrobial effect has been used as a part of cell proliferation, anti-biofilm, and antiinfection therapies in a wound-healing agent [115]. Ben et al. discovered that, with the application of MicroSafe ${ }^{\circledR}$ as an installation fluid with novel foam dressing and adverse pressure wound treatment for the patient, the wound showed significant improvement after three days of therapy $[106,107]$. In addition, $\mathrm{HOCl}$ has been reported to have antiinflammation and anti-itch effects on patients with atopic dermatitis [113]. Tomoki et al. 
reported that NC/Nga mice reduced scratching and lesions behavior and inflammatory cytokines (IL-12) after treatment of $\mathrm{HOCl}$ gel.

Microorganisms related to hand hygiene and healthcare include E. coli, Pseudomonas aeruginosa, S. aureus, Enterococcus hirae, and Candida albicans. Sipahi et al. found that StAEW was significantly effective against test microorganism (E. hirae, C. albicans, and P. aeruginosa) populations, which all decreased by $100 \%$ in 1 min treatment [116]. Available chlorine concentration (ACC), used explicitly for hand sanitizers, is effective at $30 \mathrm{ppm}$ to $150 \mathrm{ppm}$. SAEW may be a new clinical disinfectant that might be considered as a substitute for traditional alcohol-based hand sanitizer. Hsieh reported that electrolyzed oxidizing water (HOCl: $0.0125 \%$ ) showed $>99.9 \%$ antimicrobial effectiveness against Streptococcus mutans in 0.5 min treatment with little toxicity [114]. There is a possibility for electrolyzed water to be an alternative mouthwash for dental care and oral hygiene.

\section{Role of EW in Functional Food Development}

There is growing demand worldwide to produce and consume low-risk fresh food such as germinated food. Germination has widely been used to produce sprouts and young edible seeds for daily consumption. It can provide improved nutritional value, which includes free amino acids, vitamins, minerals, dietary fiber, polyphenols, and bioactive compounds, through simple germination procedures without long-term growth [23]. Seed sprouts, such as broccoli, buckwheat, radish, alfalfa, mung bean, and pea seeds, are commonly consumed raw in sandwiches and salads due to their nutritional value [117]. However, seed sprouts could easily be contaminated with foodborne pathogens, particularly E. coli O157:H7 and Salmonella, and other Shiga-toxin producing E. coli, L. monocytogenes, and B. cereus [118]. EW has become an acclaimed alternative for harsh chemical sanitizers in sprout growth and final products due to its substantial bactericidal effects, environmentally friendly attributes, and being generally recognized as having a safe (GRAS) status [22]. In general, the effect of EW applied in sprouts can be divided into three parts: inhibition of microbial growth, accumulation of functional components, and effect on morphological characteristics of sprouts.

Microbial contamination can be easily found in the process of germination due to its healthy, moist, and warm environment. In order to control the outbreak of foodborne diseases caused by a variety of pathogens in sprouts, researchers have recently found EW as a chemically based seed disinfection technique. As shown in Table 6, the total (aerobic) bacteria, molds, and yeasts on sprouts in the soaking or spraying treatment of EW after about one week's germination was generally a 1-3 log reduction compared to tap water treatment. In addition, EW could reduce the population of inoculated pathogens on vegetable sprouts to an acceptable level, such as E. coli O157:H7, L. monocytogenes, and S. enteritidis $[119,120]$. Furthermore, researchers reported that EW with increasing ACC used in seed soaking and pre-germination resulted in more significant reductions in Enterobacteriaceae populations [22]. SAEW with near-neutral $\mathrm{pH}(5-6.5)$ had excellent antibacterial effect. SAEW is more widely used in food disinfection. SAEW has been considered a useful method for eliminating pathogens with little effect on seed germination [22,44]. Meanwhile, a few authors emphasized the importance of $\mathrm{pH}$ in the germination process. The $\mathrm{pH}$ of EW can not only affect and differentiate the form of ACC, which would change antibacterial efficacy, but also affect the morphological characteristics of the sprouts. 
Table 6. Application of EW in germinated seeds.

\begin{tabular}{|c|c|c|c|c|c|c|c|c|c|}
\hline Types of se & $\begin{array}{l}\text { Types of } \\
\text { EW }\end{array}$ & ACC & $\mathrm{pH}$ & ORP & $\begin{array}{l}\text { Disinfection } \\
\text { Condition }\end{array}$ & $\begin{array}{c}\text { Reduction } \\
(\log \text { CFU/g) }\end{array}$ & $\begin{array}{l}\text { Length, Fresh Weight } \\
\text { and Nutrition Effect }\end{array}$ & $\begin{array}{l}\text { Germination } \\
\text { Time (Day) }\end{array}$ & Reference \\
\hline Alfalfa & SAEW & 45 & 5.7 & 863 & $\begin{array}{l}\text { Enterobacteriaceae } \\
\text { counts }\end{array}$ & 1.50 & - & - & [22] \\
\hline Buckwheat & SAEW & 10.94 & - & 5.05 & - & - & $\begin{array}{c}\text { Total phenolic content: } \\
32.27 \mathrm{~g} / \mathrm{kg} \\
\text { Total flavonoid content: } \\
23.97 \mathrm{~g} / \mathrm{kg} \\
\text { Rutin content: } 19.96 \mathrm{~g} / \mathrm{kg} \\
\text { Quercetin content } \\
>1.0 \mathrm{~g} / \mathrm{kg}\end{array}$ & 9 & [26] \\
\hline Buckwheat & SAEW & 28 & 6.05 & 845 & $\begin{array}{l}\text { Moulds and } \\
\text { yeasts } \\
\text { total bacteria }\end{array}$ & $\begin{array}{l}2.06 \\
0.91\end{array}$ & $\begin{array}{l}\text { No adverse effect on the } \\
\text { water germination }\end{array}$ & 8 & [44] \\
\hline Buckwheat & SAEW & 10.94 & 5.05 & - & - & - & 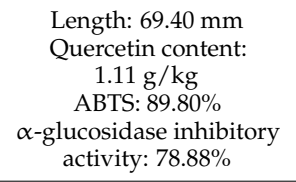 & 7 & [121] \\
\hline Buck-wheat & SAEW & 20.3 & 5.83 & - & Total bacterial & 1.12 & $\begin{array}{l}\text { Length: } 73.36 \mathrm{~mm} \\
\text { GABA contents: } \\
143.20 \mathrm{mg} / 100 \mathrm{~g} \\
\text { Rutin contents: } \\
739.9 \mathrm{mg} / 100 \mathrm{~g}\end{array}$ & 8 & [122] \\
\hline Brown rice & SAEW & 100 & $5.92-6.04$ & $938-970$ & B. cereus & 3.61 & Sprout length: $5.25 \mathrm{~mm}$ & 1 & [123] \\
\hline Triticale malt & SAEW & 30.14 & 5.32 & 977 & - & - & $\begin{array}{c}\text { a-amylase activity: } \\
0.11 \text { units } / \mathrm{mg} \text { protein } \\
\text { Phytase activity: } \\
1.13 \times 10^{-4} \text { units } / \mathrm{mg} \\
\text { protein } \\
\text { Lipase activity: } \\
0.49 \text { units } / \mathrm{mg} \text { protein }\end{array}$ & 4 & [124] \\
\hline Buckwheat & SAEW & 19.94 & 5.71 & - & - & - & $\begin{array}{l}\text { Length: } 81.52 \mathrm{~mm} \\
\text { Weight: } 8.08 \mathrm{~g} \\
\text { GABA content: } \\
\text { 55.7-113.5 mg/100 g }\end{array}$ & 9 & [125] \\
\hline Broccoli & SAEW & 40.8 & 5.47 & - & $\begin{array}{l}\text { Total bacterial } \\
\text { count }\end{array}$ & $\begin{array}{l}\text { Reduced to } \\
<6.5 \log \\
\text { CFU/g }\end{array}$ & $\begin{array}{c}\text { Sulforaphane content: } \\
96 \% \\
\text { increased compared to } \\
\text { tap water } \\
\text { Glucoerucin content: } \\
32.11 \mu \mathrm{mol} / \mathrm{g}\end{array}$ & 8 & [126] \\
\hline Broccoli & SAEW & 50 & 5.52 & - & - & - & $\begin{array}{c}\text { Sulforaphane: } \\
11.49 \mathrm{mg} / \mathrm{g} \text { in dry weight } \\
\text { Glucosinolates content: } \\
142.25 \mu \mathrm{mol} / \mathrm{g} \text { in dry } \\
\text { weight }\end{array}$ & 8 & [127] \\
\hline Mung bean & AEW & 46.09 & - & 4.47 & $\begin{array}{l}\text { Total bacterial } \\
\text { Coliform } \\
\text { Yeast and mold }\end{array}$ & $\begin{array}{l}1.23 \\
1.42 \\
1.25\end{array}$ & - & - & [128] \\
\hline Radish & EW & 50 & - & 6.5 & $\begin{array}{l}\text { Total aerobic } \\
\text { bacteria } \\
\text { Yeast and mold }\end{array}$ & $\begin{array}{l}1.39 \\
1.58\end{array}$ & $\begin{array}{l}\text { Length: } 12.41 \mathrm{~cm} \\
\text { Weight: } 13.89 \mathrm{~g}\end{array}$ & 7 & [129] \\
\hline Brown millet & SAEW & 30.35 & 834 & 5.96 & $\begin{array}{l}\text { Total bacterial } \\
\text { counts }\end{array}$ & 0.91 & $\begin{array}{l}\text { Length: } 2.2 \mathrm{~cm} \\
\text { GABA content: } \\
30.9 \mathrm{mg} / 100 \mathrm{~g} \\
\end{array}$ & 3 & [130] \\
\hline Mung bean & SAEW & 18.87 & - & 5.79 & $\begin{array}{l}\text { Total bacterial } \\
\text { counts }\end{array}$ & 1.06 & $\begin{array}{c}\text { Length }>8 \mathrm{~cm} \\
\text { Catalase activity: } 10.37 \%\end{array}$ & 6.5 & [131] \\
\hline Buckwheat & SAEW & 40 & - & 5.0 & Total bacterial & $>1$ & $\begin{array}{c}\text { Fresh weight: } 25.96 \mathrm{~g} \\
\text { Day weight: } 0.23 \mathrm{~g} \\
\text { Rutin content: } \\
42.50 \mathrm{mg} / \mathrm{g}\end{array}$ & 7 & [132] \\
\hline
\end{tabular}

Notes: AEW: acidic electrolyzed water; SAEW: slightly acidic electrolyzed water; EW: electrolyzed water. ACC: available chlorine concentration.

Sprouts' growth conditions could be directly reflected by their morphological characteristics of germination rate, hundred-grain weight, sprouts length, hypocotyl length, and hypocotyl diameter. Spraying them with EW during germination has different effects on these indexes, varying with different seed species. Specifically, SAEW could promote 
the growth of mung bean sprouts and brown rice [131,133]. However, Han reported no significant difference between SAEW treatment and control in the above-mentioned parameters [122].

Sprouts have been recognized as wellness and health-promoting foods due to their high nutrient content and bioactive compounds, including vitamins, minerals, phenolic, carotenoids, and $\gamma$-aminobutyric acid (GABA) [134-136]. The synthesis of such second metabolites often occurs when plants are stressed by various elicitors or signal molecules such as EW. Secondary metabolites play an important role in overcoming stress conditions and adapting plants to the environment [137]. SAEW has been considered a valuable means for increasing the accumulation of GABA in the millet, which increased the content of GABA higher $21 \%$ than tap water [130]. Hao reported that buckwheat treated with EW (ACC: $10.94 \mathrm{ppm}$ ) increased total phenolics, total flavonoids, and rutin content on the ninth day showing $63.0 \%, 113.07 \%$, and $128.1 \%$ increases with respect to tap water [121]. In addition, Li reported that a significantly higher content of sulforaphane (95\%) in broccoli sprouts was revealed when SAEW (40.8 ppm) was exerted, which is 1.95 times as much as tap water [126].

\section{Future Trends on the Application of EW}

As a novel promising sanitizer and cleaner, EW has become popular in the food industry in recent years. EW is widely used in various fields, including agriculture, food sanitation, livestock management, medical disinfection, clinical, and other fields using antibacterial technology. EW has been approved by Russia, Japan, and China as a sanitizing agent in the food field. Recently, the commercialization of EW generators continues to grow around the globe. Given the importance of EW, many companies have designed and produced EW generators. Examples of such companies include Eco Tree, Emcao Tech Athena, TECH Corporation, Jing Jun, and RVD Corporation, which are based in China, Japan, South Korea, and U.S.A. The companies are making electrolyzed water generators used in research laboratories, and commercialization for convenient household usage is encouraged by these companies. More importantly, with the development of portable EW generators, EW can be used for households (spray form) in the next step.

The United States Department of Agriculture National Organic Program approved $\mathrm{HOCl}$ generated by electrolyzed water as an organic-compatible sanitizer. Furthermore, EW can disinfect medical instruments, clinical environments, and object surfaces and is used for disinfecting hands, skin, and mucous membranes in China. The demand for EW products is driving many companies to search for new locations to setup facilities for manufacture, including Microcyn ${ }^{\circledR}$, Clortech $^{\circledR}$, Avenova $^{\circledR}$, Ecasol $^{\mathrm{TM}}$, and MicroSafe ${ }^{\circledR}$. Industries claim to generate EW-based products that are safe to use around the nose, mouth, and eyes while having a remarkable antimicrobial effect. Due to the high impact that it could have in breaking the chain of infection in the dental office, EW could be used in the pre-procedural stage of oral rinsing as a pre-procedural oral rinse, which has the potential to dramatically reduce the quantity of viruses, along with preventative measures and further application as personal protective equipment. Recently, in order to further improve the antibacterial activity of EW, hurdle technology, combined with two or more scaffold techniques, has been developed and it shows synergistic effects in terms of decreasing microbial population, prolonging product's shelf life, and maintaining product's quality), integrating disinfection technologies and disinfection mechanisms of EW to develop a hurdle method with synergistic effects for further improving the antimicrobial effect. The strategy will continue depending on production and manipulation, including using powerful tools (flow cytometer) and molecular biology (including transcriptomics, proteomics, metabolomics, and other omics) to fully understand the sterilization mechanism. An advanced and dynamic EW production system that is capable of overcoming all the current limitations can be overcome by conducting further research in the field.

Based on recent updates, it is believed that EW may be applied in the food industry as a possible disinfectant. EW has been certified by U.S.A. government regulators as a safer 
alternative to hazardous chemicals when applied at home, industry, and institutions, and they are mainly green and sustainable. Despite the application of EW in various sectors in Russia and Japan for several decades, its affirmation in the U.S.A. and other countries is slowly growing. Recent observations showed that there is widespread commercialization of EW around the globe. Several industries have emerged due to the importance of electrolysis water and due to rising demand. Examples of the type of company include Envirolyte, KEWS, RVD Corporation, EcoLogic Solutions Inc., and Viking pure, which are based in Asia (China, South Korea, and Japan), Europe (Latvia), and the United States.

The major advantages of EW in the growing industrial sector include the manufacture of $100 \%$ organic, non-toxic, and low-cost disinfectants for both household and industrial applications. A US-based company (EAU Technologies Inc.) completed a successful field trial in 2009 by using electrolysis water technology for Clean-in-Place (CIP) application fields, which can be applied by using Empowered Water, a brand-name for electrolysis water made by Electric Aquagenics Unlimited (EAU) Technologies. Specifically, in the industrial food sector, CIP implementations and framework enterprises clean equipment on stationary platforms (EAU 2009). Globally, depending on the location, people have differing views on sanitizing applications and electrolysis water regulations. Due to the effects of amino acid inactivation, European Union members may only apply electrolysis water to drinking water, and it is not approved for use in food products such as meat and fish. However, in the United States, it is typically applied for sanitization and disinfecting agents. United States Department of Agriculture has recently approved electrolysis to fresh produce (organic) products. Although it is simple in formulation, EW solution loses its effectiveness once it becomes exposed to water. Even though the eyes are exposed to water, humans are not affected by exposure to electrolysis as long as it is not ingested orally. The expertise of electrolysis is not widespread in the chemical industry; thus, additional exposure to this topic is required. EW is likely to be used in food plants over the next decade due to its advantages.

\section{Conclusions}

The application of EW has efficient antimicrobial with bactericidal effects on fruits, vegetables, fish, aquatic, poultry, utensils, and raw meat in various industries. However, EW with high acidity ( $\mathrm{pH}$ 2.2-2.7) and corrosive nature alters the organoleptic properties of specific foods, which limits its use. Some of these concerns have been addressed, and they alter the properties of electrolysis water, such as SAEW with $\mathrm{pH}$ (5.0-6.5). In terms of efficient microbial reduction with increasing shelf life and maintaining the nutritional and organoleptic properties of food, this can be achieved with hurdle technology (combination of EW with sonication, organic acid, and heat treatment), which overcomes disadvantage in the EW treatment process. Furthermore, the efficiency of EW depends on storage temperature, ACC and ORP, hydrogen ion concentration $(\mathrm{pH})$, salt concentration, and other physiochemical properties of EW. Furthermore, the amount of organic matter and water hardness influences its bactericidal action.

As a result, further research in this specific sector has to be improved in order to overcome limitations in the dynamic EW production system. These may include procedures for increasing the application of EW in food processing settings and further use in HACCP and Sanitation Standard Operating Procedures (SSOPs). Nowadays, SAEW has no irritation in human application and no safety issues from $\mathrm{Cl}_{2}$ off-gassing. The future of EW technology application in clinical applications appears to be very promising, especially with respect to hands, skin, and mucous membranes.

Author Contributions: P.Y.: writing and original draft preparation; R.C. and K.h.J.: revision and formatting; D.H.O.: supervision and conceptualization. All authors have read and agreed to the published version of the manuscript. 
Funding: This research study was funded by the National Research Foundation of Korea (N.R.F.), grant number (2018007551), and Brain Korea (B.K.) 21 Plus Project (Grant No. 4299990913942).

Conflicts of Interest: The authors declare no conflict of interest.

\section{References}

1. Aworh, O.C. Food safety issues in fresh produce supply chain with particular reference to sub-Saharan Africa. Food Control 2021, 123, 107737. [CrossRef]

2. Fung, F.; Wang, H.S.; Menon, S. Food safety in the 21st century. Biomed. J. 2018, 41, 88-95. [CrossRef] [PubMed]

3. Lee, H.; Yoon, Y. Etiological Agents Implicated in Foodborne Illness World Wide. Food Sci. Anim. Resour. 2021, 41, 1-7. [CrossRef]

4. Grace, D.; Wu, F.; Havelaar, A.H. MILK Symposium review: Foodborne diseases from milk and milk products in developing countries-Review of causes and health and economic implications. J. Dairy Sci. 2020, 103, 9715-9729. [CrossRef]

5. World Health Organization. WHO Estimates of the Global Burden of Foodborne Diseases: Foodborne Disease Burden epidemiology Reference Group 2007-2015. Available online: https://apps.who.int/iris/handle/10665/199350 (accessed on 1 October 2021).

6. Hoffmann, S.; Scallan, E. Epidemiology, cost, and risk analysis of foodborne disease. In Foodborne Diseases, 3rd ed.; Dodd, C.E.R., Aldsworth, T., Stein, R.A., Cliver, D.O., Riemann, H.P., Eds.; Elsevier: Amsterdam, The Netherlands, 2017 ; pp. 31-63.

7. Sedrakyan, A.M.; Ktsoyan, Z.A.; Arakelova, K.A.; Zakharyan, M.K.; Hovhannisyan, A.I.; Gevorgyan, Z.U.; Mnatsakanyan, A.A.; Kakabadze, E.G.; Makalatia, K.B.; Chanishvili, N.A.; et al. Extended-Spectrum beta-Lactamases in Human Isolates of Multidrug-Resistant Non-typhoidal Salmonella enterica. Front. Microbiol. 2020, 11, 592223. [CrossRef] [PubMed]

8. Byun, K.H.; Han, S.H.; Yoon, J.W.; Park, S.H.; Ha, S.D. Efficacy of chlorine-based disinfectants (sodium hypochlorite and chlorine dioxide) on Salmonella Enteritidis planktonic cells, biofilms on food contact surfaces and chicken skin. Food Control 2021, 123, 107838. [CrossRef]

9. Brodowska, A.J.; Nowak, A.; Smigielski, K. Ozone in the food industry: Principles of ozone treatment, mechanisms of action, and applications: An overview. Crit. Rev. Food Sci. Nutr. 2018, 58, 2176-2201. [CrossRef] [PubMed]

10. Moretro, T.; Fanebust, H.; Fagerlund, A.; Langsrud, S. Whole room disinfection with hydrogen peroxide mist to control Listeria monocytogenes in food industry related environments. Int. J. Food Microbiol. 2019, 292, 118-125. [CrossRef]

11. Korany, A.M.; Hua, Z.; Green, T.; Hanrahan, I.; El-Shinawy, S.H.; El-Kholy, A.; Hassan, G.; Zhu, M.J. Efficacy of Ozonated Water, Chlorine, Chlorine Dioxide, Quaternary Ammonium Compounds and Peroxyacetic Acid Against Listeria monocytogenes Biofilm on Polystyrene Surfaces. Front. Microbiol. 2018, 9, 2296. [CrossRef]

12. Ju, J.; Xie, Y.; Guo, Y.; Cheng, Y.; Qian, H.; Yao, W. Application of edible coating with essential oil in food preservation. Crit. Rev. Food Sci. Nutr. 2019, 59, 2467-2480. [CrossRef] [PubMed]

13. Zhao, L.; Li, S.; Yang, H. Recent advances on research of electrolyzed water and its applications. Curr. Opin. Food Sci. 2021, 41, 180-188. [CrossRef]

14. Yan, P.; Daliri, E.B.; Oh, D.H. New Clinical Applications of Electrolyzed Water: A Review. Microorganisms 2021, 9, 136. [CrossRef] [PubMed]

15. Aider, M.; Gnatko, E.; Benali, M.; Plutakhin, G.; Kastyuchik, A. Electro-activated aqueous solutions: Theory and application in the food industry and biotechnology. Innov. Food Sci. Emerg. Technol. 2012, 15, 38-49. [CrossRef]

16. Zheng, W.; Kang, R.; Wang, H.; Li, B.; Xu, C.; Wang, S. Airborne bacterial reduction by spraying slightly acidic electrolyzed water in a laying-hen house. J. Air Waste Manag. Assoc. 2013, 63, 1205-1211. [CrossRef]

17. Phuvasate, S.; Su, Y.C. Effects of electrolyzed oxidizing water and ice treatments on reducing histamine-producing bacteria on fish skin and food contact surface. Food Control 2010, 21, 286-291. [CrossRef]

18. Possas, A.; Perez-Rodriguez, F.; Tarlak, F.; Garcia-Gimeno, R.M. Quantifying and modelling the inactivation of Listeria monocytogenes by electrolyzed water on food contact surfaces. J. Food Eng. 2021, 290, 110287. [CrossRef]

19. Pangloli, P.; Hung, Y.C. Efficacy of slightly acidic electrolyzed water in killing or reducing Escherichia coli O157:H7 on iceberg lettuce and tomatoes under simulated food service operation conditions. J. Food Sci. 2011, 76, M361-M366. [CrossRef]

20. Afari, G.K.; Hung, Y.C. A meta-analysis on the effectiveness of electrolyzed water treatments in reducing foodborne pathogens on different foods. Food Control 2018, 93, 150-164. [CrossRef]

21. He, Y.; Zhao, X.; Chen, L.; Zhao, L.; Yang, H.S. Effect of electrolysed water generated by sodium chloride combined with sodium bicarbonate solution against Listeria innocua in broth and on shrimp. Food Control 2021, 127, 108134. [CrossRef]

22. Zhang, C.; Zhao, Z.; Yang, G.; Shi, Y.; Zhang, Y.; Shi, C.; Xia, X. Effect of slightly acidic electrolyzed water on natural Enterobacteriaceae reduction and seed germination in the production of alfalfa sprouts. Food Microbiol. 2021, 97, 103414. [CrossRef] [PubMed]

23. Zhang, C.L.; Zhang, Y.Y.; Zhao, Z.Y.; Liu, W.F.; Chen, Y.Q.; Yang, G.J.; Xia, X.D.; Cao, Y.F. The application of slightly acidic electrolyzed water in pea sprout production to ensure food safety, biological and nutritional quality of the sprout. Food Control 2019, 104, 83-90. [CrossRef]

24. Fransisca, L.; Zhou, B.; Park, H.; Feng, H. The effect of calcinated calcium and chlorine treatments on Escherichia coli O157:H7 87-23 population reduction in radish sprouts. J. Food Sci. 2011, 76, M404-M412. [CrossRef] 
25. Lu, Z.H.; Zhang, Y.; Li, L.T.; Curtis, R.B.; Kong, X.L.; Fulcher, R.G.; Zhang, G.; Cao, W. Inhibition of microbial growth and enrichment of gamma-aminobutyric acid during germination of brown rice by electrolyzed oxidizing water. J. Food Prot. 2010, 73, 483-487. [CrossRef]

26. Hao, J.; Wang, Q. Application of electrolyzed water in fruits and vegetables industry. In Electrolyzed Water in Food: Fundamentals and Applications; Ding, T., Oh, D.H., Liu, D., Eds.; Springer: Singapore, 2019; pp. 67-111.

27. Pou, K.R.J.; Raghavan, V. Recent Advances in the Application of High Pressure Processing-Based Hurdle Approach for Enhancement of Food Safety and Quality. J. Biosyst. Eng. 2020, 45, 175-187. [CrossRef]

28. Jo, H.Y.; Tango, C.N.; Oh, D.H. Influence of different organic materials on chlorine concentration and sanitization of slightly acidic electrolyzed water. Lebensm. Wiss. Technol. 2018, 92, 187-194. [CrossRef]

29. Deng, L.Z.; Mujumdar, A.S.; Pan, Z.; Vidyarthi, S.K.; Xu, J.; Zielinska, M.; Xiao, H.W. Emerging chemical and physical disinfection technologies of fruits and vegetables: A comprehensive review. Crit. Rev. Food Sci. Nutr. 2020, 60, 2481-2508. [CrossRef]

30. Akbulut, M.B.; Eldeniz, A.Ü. In vitro antimicrobial activity of different electrochemicallyactivated solutions on enterococcus faecalis. Eur. Oral Res. 2019, 53, 44-50. [CrossRef]

31. Fukuzaki, S. Mechanisms of actions of sodium hypochlorite in cleaning and disinfection processes. Biocontrol Sci. 2006, 11, 147-157. [CrossRef] [PubMed]

32. Cheng, X.; Tian, Y.; Zhao, C.; Qu, T.; Ma, C.; Liu, X.; Yu, Q. Bactericidal Effect of Strong Acid Electrolyzed Water against Flow Enterococcus faecalis Biofilms. J. Endod. 2016, 42, 1120-1125. [CrossRef] [PubMed]

33. Ding, T.; Xuan, X.T.; Li, J.; Chen, S.G.; Liu, D.H.; Ye, X.Q.; Shi, J.; Xue, S.J. Disinfection efficacy and mechanism of slightly acidic electrolyzed water on Staphylococcus aureus in pure culture. Food Control 2016, 60, 505-510. [CrossRef]

34. Tang, W.W.; Zeng, X.P.; Zhao, Y.S.; Ye, G.Q.; Gui, W.C.; Ni, Y.M. Disinfection Effect and Its Mechanism of Electrolyzed Oxidizing Water on Spores of Bacillus subtilis var. niger. Food Sci. Biotechnol. 2011, 20, 889-895. [CrossRef]

35. Memar, M.Y.; Ghotaslou, R.; Samiei, M.; Adibkia, K. Antimicrobial use of reactive oxygen therapy: Current insights. Infect. Drug Resist. 2018, 11, 567-576. [CrossRef]

36. Liu, Q.; Chen, L.; Laserna, A.K.C.; He, Y.; Feng, X.; Yang, H.S. Synergistic action of electrolyzed water and mild heat for enhanced microbial inactivation of Escherichia coli O157:H7 revealed by metabolomics analysis. Food Control 2020, 110, 107026. [CrossRef]

37. Liu, Q.; Wu, J.; Lim, Z.Y.; Lai, S.; Lee, N.; Yang, H. Metabolite profiling of Listeria innocua for unravelling the inactivation mechanism of electrolysed water by nuclear magnetic resonance spectroscopy. Int. J. Food Microbiol. 2018, 271, 24-32. [CrossRef]

38. Liu, R.; Yu, Z.-L.; Sun, Y.-L.; Zhou, S.-M. The enzymatic browning reaction inhibition effect of strong acidic electrolyzed water on different parts of sweet potato slices. Food Biosci. 2021, 43, 101252. [CrossRef]

39. Wu, S.; Nie, Y.; Zhao, J.; Fan, B.; Huang, X.; Li, X.; Sheng, J.; Meng, D.; Ding, Y.; Tang, X. The synergistic effects of low-concentration acidic electrolyzed water and ultrasound on the storage quality of fresh-sliced button mushrooms. Food Bioprocess Technol. 2018, 11, 314-323. [CrossRef]

40. Li, H.; Ren, Y.; Hao, J.; Liu, H. Dual effects of acidic electrolyzed water treatments on the microbial reduction and control of enzymatic browning for fresh-cut lotus root. J. Food Saf. 2017, 37, e12333. [CrossRef]

41. Liu, W.; Wang, Q.; Mei, J.; Xie, J. Shelf-life extension of refrigerated turbot (Scophthalmus maximus) by using weakly acidic electrolyzed water and active coatings containing daphnetin emulsions. Front. Nutr. 2021, 8, 696212. [CrossRef] [PubMed]

42. Puligundla, P.; Kim, J.W.; Mok, C. Broccoli sprout washing with electrolyzed water: Effects on microbiological and physicochemical characteristics. Lebensm. Wiss. Technol. 2018, 92, 600-606. [CrossRef]

43. Hussain, M.S.; Kwon, M.; Park, E.J.; Seheli, K.; Huque, R.; Oh, D.H. Disinfection of Bacillus cereus biofilms on leafy green vegetables with slightly acidic electrolyzed water, ultrasound and mild heat. Lebensm. Wiss. Technol. 2019, 116, 108582. [CrossRef]

44. Liang, D.; Wang, Q.F.; Zhao, D.D.; Han, X.; Hao, J.X. Systematic application of slightly acidic electrolyzed water (SAEW) for natural microbial reduction of buckwheat sprouts. Lebensm. Wiss. Technol. 2019, 108, 14-20. [CrossRef]

45. Cap, M.; Rojas, D.; Fernandez, M.; Fulco, M.; Rodriguez, A.; Soteras, T.; Cristos, D.; Mozgovoj, M. Effectiveness of short exposure times to electrolyzed water in reducing Salmonella spp and Imidacloprid in lettuce. Lebensm. Wiss. Technol. 2020, 128, 109496. [CrossRef]

46. Sheng, L.N.; Shen, X.Y.; Ulloa, O.; Suslow, T.V.; Hanrahan, I.; Zhu, M.J. Evaluation of JC9450 and Neutral Electrolyzed Water in Controlling Listeria monocytogenes on Fresh Apples and Preventing Cross-Contamination. Front. Microbiol. 2020, 10, 3128. [CrossRef]

47. Ogunniyi, A.D.; Dandie, C.E.; Brunetti, G.; Drigo, B.; Aleer, S.; Hall, B.; Ferro, S.; Deo, P.; Venter, H.; Myers, B.; et al. Neutral electrolyzed oxidizing water is effective for pre-harvest decontamination of fresh produce. Food Microbiol. 2021, $93,103610$. [CrossRef]

48. Ogunniyi, A.D.; Tenzin, S.; Ferro, S.; Venter, H.; Pi, H.F.; Amorico, T.; Deo, P.; Trott, D.J. A pH-neutral electrolyzed oxidizing water significantly reduces microbial contamination of fresh spinach leaves. Food Microbiol. 2021, 93, 103614. [CrossRef] [PubMed]

49. Chen, Y.X.; Guo, X.N.; Xiang, J.J.; Sun, X.H.; Zhu, K.X. Effects of wheat tempering with slightly acidic electrolyzed water on the microbial, biological, and chemical characteristics of different flour streams. Lebensm. Wiss. Technol. 2020, 118, 108790. [CrossRef]

50. Huang, L.C.; Luo, X.; Gao, J.W.; Matthews, K.R. Influence of water antimicrobials and storage conditions on inactivating MS2 bacteriophage on strawberries. Int. J. Food Microbiol. 2019, 291, 67-71. [CrossRef]

51. Sheng, L.N.; Shen, X.Y.; Zhu, M.J. Screening of non-pathogenic surrogates of Listeria monocytogenes applicable for chemical antimicrobial interventions of fresh apples. Food Control 2020, 110, 106977. [CrossRef] 
52. Zhang, C.L.; Cao, W.; Hung, Y.C.; Li, B.M. Disinfection effect of slightly acidic electrolyzed water on celery and cilantro. Food Control 2016, 69, 147-152. [CrossRef]

53. Song, H.; Lee, J.Y.; Lee, H.W.; Ha, J.H. Inactivation of bacteria causing soft rot disease in fresh cut cabbage using slightly acidic electrolyzed water. Food Control 2021, 128, 108217. [CrossRef]

54. Tang, J.Y.; Chen, H.B.; Lin, H.T.; Hung, Y.C.; Xie, H.L.; Chen, Y.H. Acidic electrolyzed water treatment delayed fruit disease development of harvested longans through inducing the disease resistance and maintaining the ROS metabolism systems. Postharvest Biol. Technol. 2021, 171, 111349. [CrossRef]

55. Chen, H.Q.; Zhong, Q.X. Antibacterial activity of acidified sodium benzoate against Escherichia coli O157:H7, Salmonella enterica, and Listeria monocytogenes in tryptic soy broth and on cherry tomatoes. Int. J. Food Microbiol. 2018, 274, 38-44. [CrossRef] [PubMed]

56. Abadias, M.; Usall, J.; Oliveira, M.; Alegre, I.; Vinas, I. Efficacy of neutral electrolyzed water (NEW) for reducing microbial contamination on minimally-processed vegetables. Int. J. Food Microbiol. 2008, 123, 151-158. [CrossRef] [PubMed]

57. Rahman, S.M.E.; Khan, I.; Oh, D.H. Electrolyzed Water as a Novel Sanitizer in the Food Industry: Current Trends and Future Perspectives. Compr. Rev. Food Sci. Food Saf. 2016, 15, 471-490. [CrossRef] [PubMed]

58. Raybaudi-Massilia, R.M.; Mosqueda-Melgar, J.; Soliva-Fortuny, R.; Martin-Belloso, O. Control of Pathogenic and Spoilage Microorganisms in Fresh-cut Fruits and Fruit Juices by Traditional and Alternative Natural Antimicrobials. Compr. Rev. Food Sci. Food Saf. 2009, 8, 157-180. [CrossRef]

59. Estell, M.; Hughes, J.; Grafenauer, S. Plant Protein and Plant-Based Meat Alternatives: Consumer and Nutrition Professional Attitudes and Perceptions. Sustainability 2021, 13, 1478. [CrossRef]

60. Orejel, J.R.C.; Cano-Buendia, J.A. Applications of Electrolyzed Water as a Sanitizer in the Food and Animal-By Products Industry. Processes 2020, 8, 534. [CrossRef]

61. Northcutt, J.; Smith, D.; Ingram, K.D.; Hinton, A.; Musgrove, M. Recovery of Bacteria from Broiler Carcasses after Spray Washing with Acidified Electrolyzed Water or Sodium Hypochlorite Solutions. Poult. Sci. 2007, 86, 2239-2244. [CrossRef] [PubMed]

62. Wang, H.H.; Qi, J.; Duan, D.B.; Dong, Y.; Xu, X.L.; Zhou, G.Z. Combination of a novel designed spray cabinet and electrolyzed water to reduce microorganisms on chicken carcasses. Food Control 2018, 86, 200-206. [CrossRef]

63. Al-Holy, M.A.; Rasco, B.A. The bactericidal activity of acidic electrolyzed oxidizing water against Escherichia coli O157:H7, Salmonella Typhimurium, and Listeria monocytogenes on raw fish, chicken and beef surfaces. Food Control 2015, 54, 317-321. [CrossRef]

64. Han, D.; Hung, Y.C.; Wang, L.X. Evaluation of the antimicrobial efficacy of neutral electrolyzed water on pork products and the formation of viable but nonculturable (VBNC) pathogens. Food Microbiol. 2018, 73, 227-236. [CrossRef]

65. Rahman, S.M.E.; Park, J.; Song, K.B.; Al-Harbi, N.A.; Oh, D.H. Effects of Slightly Acidic Low Concentration Electrolyzed Water on Microbiological, Physicochemical, and Sensory Quality of Fresh Chicken Breast Meat. J. Food Sci. 2012, 77, M35-M41. [CrossRef]

66. Medina-Gudiño, J.; Rivera-Garcia, A.; Santos-Ferro, L.; Ramirez-Orejel, J.C.; Agredano-Moreno, L.T.; Jimenez-Garcia, L.F.; PaezEsquiliano, D.; Martinez-Vidal, S.; Andrade-Esquivel, E.; Cano-Buendia, J.A. Analysis of Neutral Electrolyzed Water anti-bacterial activity on contaminated eggshells with Salmonella enterica or Escherichia coli. Int. J. Food Microbiol. 2020, 320, 108538. [CrossRef] [PubMed]

67. Sheng, X.W.; Shu, D.Q.; Tang, X.J.; Zang, Y.T. Effects of slightly acidic electrolyzed water on the microbial quality and shelf life extension of beef during refrigeration. Food Sci. Nutr. 2018, 6, 1975-1981. [CrossRef] [PubMed]

68. Veasey, S.; Muriana, P.M. Evaluation of Electrolytically-Generated Hypochlorous Acid ("Electrolyzed Water") for Sanitation of Meat and Meat-Contact Surfaces. Foods 2016, 5, 42. [CrossRef]

69. Hernandez-Pimentel, V.M.; Regalado-Gonzalez, C.; Nava-Morales, G.M.; Meas-Vong, Y.; Castaneda-Serrano, M.P.; GarciaAlmendarez, B.E. Effect of neutral electrolyzed water as antimicrobial intervention treatment of chicken meat and on trihalomethanes formation. J. Appl. Poult. Res. 2020, 29, 622-635. [CrossRef]

70. Brychcy, E.; Malik, M.; Drozdzewski, P.; Ulbin-Figlewicz, N.; Jarmoluk, A. Low-concentrated acidic electrolysed water treatment of pork: Inactivation of surface microbiota and changes in product quality. Int. J. Food Sci. Technol. 2015, 50, 2340-2350. [CrossRef]

71. Arya, R.; Bryant, M.; Degala, H.L.; Mahapatra, A.K.; Kannan, G. Effectiveness of a low-cost household electrolyzed water generator in reducing the populations of Escherichia coli K12 on inoculated beef, chevon, and pork surfaces. J. Food Process. Preserv. 2018, 42, e13636. [CrossRef]

72. Botta, C.; Ferrocino, I.; Cavallero, M.C.; Riva, S.; Giordano, M.; Cocolin, L. Potentially active spoilage bacteria community during the storage of vacuum packaged beefsteaks treated with aqueous ozone and electrolyzed water. Int. J. Food Microbiol. 2018, 266, 337-345. [CrossRef] [PubMed]

73. Torres-Rosales, E.; Rivera-Garcia, A.; Rosario-Perez, P.J.; Ramirez-Orejel, J.C.; Paez-Esquiliano, D.; Martinez-Vidal, S.; GuzmanOlea, E.; Cano-Buendia, J.A. Application of Neutral Electrolyzed Water on pork chops and its impact on meat quality. Sci. Rep. 2020, 10, 19910. [CrossRef]

74. Sun, J.; Jiang, X.; Chen, Y.; Lin, M.; Tang, J.; Lin, Q.; Fang, L.; Li, M.; Hung, Y.-C.; Lin, H. Recent trends and applications of electrolyzed oxidizing water in fresh foodstuff preservation and safety control. Food Chem. 2022, 369, 130873. [CrossRef] [PubMed]

75. Al-Qadiri, H.M.; Al-Holy, M.A.; Shiroodi, S.G.; Ovissipour, M.; Govindan, B.N.; Al-Alami, N.; Sablani, S.S.; Rasco, B. Effect of acidic electrolyzed water-induced bacterial inhibition and injury in live clam (Venerupis philippinarum) and mussel (Mytilus edulis). Int. J. Food Microbiol. 2016, 231, 48-53. [CrossRef] [PubMed] 
76. Xuan, X.-T.; Fan, Y.-F.; Ling, J.-G.; Hu, Y.-Q.; Liu, D.-H.; Chen, S.-G.; Ye, X.-Q.; Ding, T. Preservation of squid by slightly acidic electrolyzed water ice. Food Control 2017, 73, 1483-1489. [CrossRef]

77. Zhao, L.; Zhang, Z.; Wang, M.; Sun, J.; Li, H.; Malakar, P.K.; Liu, H.; Pan, Y.; Zhao, Y. New insights into the changes of the proteome and microbiome of shrimp (litopenaeus vannamei) stored in acidic electrolyzed water ice. J. Agric. Food Chem. 2018, 66, 4966-4976. [CrossRef]

78. Jung, S.; Ko, B.S.; Jang, H.-J.; Park, H.J.; Oh, S.-W. Effects of slightly acidic electrolyzed water ice and grapefruit seed extract ice on shelf life of brown sole (Pleuronectes herzensteini). Food Sci. Biotechnol. 2018, 27, 261-267. [CrossRef]

79. Yan, W.; Zhang, Y.; Yang, R.; Zhao, W. Combined effect of slightly acidic electrolyzed water and ascorbic acid to improve quality of whole chilled freshwater prawn (Macrobrachium rosenbergii). Food Control 2020, 108, 106820. [CrossRef]

80. Tantratian, S.; Kaephen, K. Shelf-life of shucked oyster in epigallocatechin-3-gallate with slightly acidic electrolyzed water washing under refrigeration temperature. LWT 2020, 118, 108733. [CrossRef]

81. Liu, D.; Lv, R. Safety evaluation of electrolyzed water. In Electrolyzed Water in Food: Fundamentals and Applications; Ding, T., Oh, D.H., Liu, D., Eds.; Springer: Singapore, 2019; pp. 261-267.

82. Hricova, D.; Stephan, R.; Zweifel, C. Electrolyzed water and its application in the food industry. J. Food Prot. 2008, 71, 1934-1947. [CrossRef]

83. Khan, I.; Tango, C.N.; Miskeen, S.; Lee, B.H.; Oh, D.H. Hurdle technology: A novel approach for enhanced food quality and safety-A review. Food Control 2017, 73, 1426-1444. [CrossRef]

84. Mohammad, Z.; Kalbasi-Ashtari, A.; Riskowski, G.; Juneja, V.; Castillo, A. Inactivation of Salmonella and Shiga toxin-producing Escherichia coli (STEC) from the surface of alfalfa seeds and sprouts by combined antimicrobial treatments using ozone and electrolyzed water. Food Res. Int. 2020, 136, 109488. [CrossRef]

85. Zhao, L.; Zhao, M.Y.; Phey, C.P.; Yang, H.S. Efficacy of low concentration acidic electrolysed water and levulinic acid combination on fresh organic lettuce (Lactuca sativa Var. Crispa L.) and its antimicrobial mechanism. Food Control 2019, 101, 241-250. [CrossRef]

86. Mansur, A.R.; Tango, C.N.; Kim, G.H.; Oh, D.H. Combined effects of slightly acidic electrolyzed water and fumaric acid on the reduction of foodborne pathogens and shelf life extension of fresh pork. Food Control 2015, 47, 277-284. [CrossRef]

87. Hussain, M.S.; Tango, C.N.; Oh, D.H. Inactivation kinetics of slightly acidic electrolyzed water combined with benzalkonium chloride and mild heat treatment on vegetative cells, spores, and biofilms of Bacillus cereus. Food Res. Int. 2019, 116, 157-167. [CrossRef]

88. Park, S.Y.; Ha, S.D. Reduction of Escherichia coli and Vibrio parahaemolyticus Counts on Freshly Sliced Shad (Konosirus punctatus) by Combined Treatment of Slightly Acidic Electrolyzed Water and Ultrasound Using Response Surface Methodology. Food Bioproc. Tech. 2015, 8, 1762-1770. [CrossRef]

89. Xuan, X.T.; Ding, T.; Li, J.; Ahn, J.H.; Zhao, Y.; Chen, S.G.; Ye, X.Q.; Liu, D.H. Estimation of growth parameters of Listeria monocytogenes after sublethal heat and slightly acidic electrolyzed water (SAEW) treatment. Food Control 2017, 71, 17-25. [CrossRef]

90. Rahman, S.M.E.; Wang, J.; Oh, D.H. Synergistic effect of low concentration electrolyzed water and calcium lactate to ensure microbial safety, shelf life and sensory quality of fresh pork. Food Control 2013, 30, 176-183. [CrossRef]

91. Cichoski, A.J.; Flores, D.R.M.; De Menezes, C.R.; Jacob-Lopes, E.; Zepka, L.Q.; Wagner, R.; Barin, J.S.; Flores, E.M.D.; Fernandes, M.D.; Campagnol, P.C.B. Ultrasound and slightly acid electrolyzed water application: An efficient combination to reduce the bacterial counts of chicken breast during pre-chilling. Int. J. Food Microbiol. 2019, 301, 27-33. [CrossRef]

92. Rahman, S.M.E.; Jin, Y.G.; Oh, D.H. Combined Effects of Alkaline Electrolyzed Water and Citric Acid with Mild Heat to Control Microorganisms on Cabbage. J. Food Sci. 2010, 75, M111-M115. [CrossRef] [PubMed]

93. Mansur, A.R.; Oh, D.H. Modeling the Growth of Epiphytic Bacteria on Kale Treated by Thermosonication Combined with Slightly Acidic Electrolyzed Water and Stored under Dynamic Temperature Conditions. J. Food Sci. 2016, 81, M2021-M2030. [CrossRef]

94. Lv, R.L.; Muhammad, A.I.; Zou, M.; Yu, Y.; Fan, L.H.; Zhou, J.W.; Ding, T.; Ye, X.Q.; Guo, M.M.; Liu, D.H. Hurdle enhancement of acidic electrolyzed water antimicrobial efficacy on Bacillus cereus spores using ultrasonication. Appl. Microbiol. Biotechnol. 2020, 104, 4505-4513. [CrossRef]

95. Jiang, Y.R.; Ai, C.M.; Liao, X.Y.; Liu, D.H.; Ding, T. Effect of slightly acidic electrolyzed water (SAEW) and ultraviolet light illumination pretreatment on microflora inactivation of coriander. Lebensm. Wiss. Technol. 2020, 132, 109898. [CrossRef]

96. Lv, R.L.; Mingming, Z.; Chen, W.J.; Wang, D.L.; Zhou, J.W.; Ding, T.; Ye, X.Q.; Liu, D.H. A hurdle approach of acidic electrolyzed water simultaneous with ultrasound to inactivate Bacillus cereus. J. Food Process. Preserv. 2019, 43, e14148. [CrossRef]

97. Shao, L.T.; Dong, Y.; Chen, X.J.; Xu, X.L.; Wang, H.H. Modeling the elimination of mature biofilms formed by Staphylococcus aureus and Salmonella spp. Using combined ultrasound and disinfectants. Ultrason. Sonochem. 2020, 69, 105269. [CrossRef] [PubMed]

98. Liu, Q.; Jin, X.X.; Feng, X.; Yang, H.S.; Fu, C.L. Inactivation kinetics of Escherichia coli O157:H7 and Salmonella Typhimurium on organic carrot (Daucus carota L.) treated with low concentration electrolyzed water combined with short-time heat treatment. Food Control 2019, 106, 106702. [CrossRef]

99. Ovissipour, M.; Shiroodi, S.G.; Rasco, B.; Tang, J.M.; Sablani, S.S. Electrolyzed water and mild-thermal processing of Atlantic salmon (Salmo salar): Reduction of Listeria monocytogenes and changes in protein structure. Int. J. Food Microbiol. 2018, 276, 10-19. [CrossRef] 
100. Du, S.P.; Zhang, Z.H.; Xiao, L.L.; Lou, Y.; Pan, Y.J.; Zhao, Y. Acidic Electrolyzed Water as a Novel Transmitting Medium for High Hydrostatic Pressure Reduction of Bacterial Loads on Shelled Fresh Shrimp. Front. Microbiol. 2016, 7, 305. [CrossRef] [PubMed]

101. Chen, J.; Xu, B.; Deng, S.G.; Huang, Y.T. Effect of Combined Pretreatment with Slightly Acidic Electrolyzed Water and Botanic Biopreservative on Quality and Shelf Life of Bombay Duck (Harpadon nehereus). J. Food Qual. 2016, 39, 116-125. [CrossRef]

102. Athayde, D.R.; Flores, D.R.M.; da Silva, J.S.; Genro, A.L.G.; Silva, M.S.; Klein, B.; Mello, R.; Campagnol, P.C.B.; Wagner, R.; de Menezes, C.R.; et al. Application of electrolyzed water for improving pork meat quality. Food Res. Int. 2017, 100, 757-763. [CrossRef]

103. Miks-Krajnik, M.; Feng, L.X.J.; Bang, W.S.; Yuk, H.G. Inactivation of Listeria monocytogenes and natural microbiota on raw salmon fillets using acidic electrolyzed water, ultraviolet light or/and ultrasounds. Food Control 2017, 74, 54-60. [CrossRef]

104. Palotas, P.; Palotas, P.; Jonas, G.; Lehel, J.; Friedrich, L. Preservative Effect of Novel Combined Treatment with Electrolyzed Active Water and Lysozyme Enzyme to Increase the Storage Life of Vacuum-Packaged Carp. J. Food Qual. 2020, 2020, 4861471. [CrossRef]

105. Tango, C.N.; Khan, I.; Kounkeu, P.F.N.; Momna, R.; Hussain, M.S.; Oh, D.H. Slightly acidic electrolyzed water combined with chemical and physical treatments to decontaminate bacteria on fresh fruits. Food Microbiol. 2017, 67, 97-105. [CrossRef]

106. Ben-Nakhi, M.E.; Eltayeb, H.I. First Middle East Experience with Novel Foam Dressing Together with Negative Pressure Wound Therapy and Instillation. Cureus 2018, 10, e3415. [CrossRef]

107. Fadriquela, A.; Sajo, M.E.J.; Bajgai, J.; Kim, D.H.; Kim, C.S.; Kim, S.K.; Lee, K.J. Effects of Strong Acidic Electrolyzed Water in Wound Healing via Inflammatory and Oxidative Stress Response. Oxid. Med. Cell. Longev. 2020, 2020, 2459826. [CrossRef]

108. Gomez-Garcia, M.; Sol, C.; de Nova, P.J.G.; Puyalto, M.; Mesas, L.; Puente, H.; Mencia-Ares, O.; Miranda, R.; Arguello, H.; Rubio, P.; et al. Antimicrobial activity of a selection of organic acids, their salts and essential oils against swine enteropathogenic bacteria. Porcine Health Manag. 2019, 5, 32. [CrossRef]

109. Rodriguez-Lopez, L.; Rincon-Fontan, M.; Vecino, X.; Cruz, J.M.; Moldes, A.B. Preservative and Irritant Capacity of Biosurfactants From Different Sources: A Comparative Study. J. Pharm. Sci. 2019, 108, 2296-2304. [CrossRef]

110. Sheng, X.W.; Bing, S.; Lu, C.Q.; Yuan, X.Y.; Zang, Y.T.; Zhan, Z.W.; Shu, D.Q.; Li, Y.J.; Li, M.T.; Wu, B.Q. A combined approach using slightly acidic electrolyzed water and UV exposure to improve egg internal quality during storage. Poult. Sci. 2020, 99, 6007-6012. [CrossRef] [PubMed]

111. Giarratana, N.; Rajan, B.; Kamala, K.; Mendenhall, M.; Reiner, G. A sprayable Acid-Oxidizing solution containing hypochlorous acid (AOS2020) efficiently and safely inactivates SARS-Cov-2: A new potential solution for upper respiratory tract hygiene. Eur. Arch. Otorhinolaryngol. 2021, 278, 3099-3103. [CrossRef]

112. Zmuda, H.M.; Mohamed, A.; Raval, Y.S.; Call, D.R.; Schuetz, A.N.; Patel, R.; Beyenal, H. Hypochlorous acid-generating electrochemical scaffold eliminates Candida albicans biofilms. J. Appl. Microbiol. 2020, 129, 776-786. [CrossRef] [PubMed]

113. Fukuyama, T.; Martel, B.C.; Linder, K.E.; Ehling, S.; Ganchingco, J.R.; Baumer, W. Hypochlorous acid is antipruritic and anti-inflammatory in a mouse model of atopic dermatitis. Clin. Exp. Allergy 2018, 48, 78-88. [CrossRef] [PubMed]

114. Hsieh, Y.L.; Yao, J.C.; Hsieh, S.C.; Teng, N.C.; Chu, Y.T.; Yu, W.X.; Chen, C.H.; Chang, L.Y.; Huang, C.S.; Lee, T.H.; et al. The In Vivo Toxicity and Antimicrobial Properties for Electrolyzed Oxidizing (EO) Water-Based Mouthwashes. Materials 2020, 13, 4299. [CrossRef] [PubMed]

115. Reis, R.; Sipahi, H.; Dinc, O.; Kavaz, T.; Charehsaz, M.; Dimoglo, A.; Aydin, A. Toxicity, mutagenicity and stability assessment of simply produced electrolyzed water as a wound healing agent in vitro. Hum. Exp. Toxicol. 2021, 40, 452-463. [CrossRef]

116. Sipahi, H.; Reis, R.; Dinc, O.; Kavaz, T.; Dimoglo, A.; Aydin, A. In vitro biocompatibility study approaches to evaluate the safety profile of electrolyzed water for skin and eye. Hum. Exp. Toxicol. 2019, 38, 1314-1326. [CrossRef]

117. Di Gioia, F.; Renna, M.; Santamaria, P. Sprouts, microgreens and "baby leaf" vegetables. In Minimally Processed Refrigerated Fruits and Vegetables. Food Engineering Series; Yildiz, F., Wiley, R., Eds.; Springer: Boston, MA, USA, 2017; pp. 403-432.

118. Chen, Y.H.; Pouillot, R.; Farakos, S.M.S.; Duret, S.; Spungen, J.; Fu, T.J.; Shakir, F.; Homola, P.A.; Dennis, S.; Van Doren, J.M. Risk Assessment of Salmonellosis from Consumption of Alfalfa Sprouts and Evaluation of the Public Health Impact of Sprout Seed Treatment and Spent Irrigation Water Testing. Risk Anal. 2018, 38, 1738-1757. [CrossRef]

119. Phua, L.K.; Neo, S.Y.; Khoo, G.H.; Yuk, H.G. Comparison of the efficacy of various sanitizers and hot water treatment in inactivating inoculated foodborne pathogens and natural microflora on mung bean sprouts. Food Control 2014, 42, 270-276. [CrossRef]

120. Ngnitcho, P.F.K.; Khan, I.; Tango, C.N.; Hussain, M.S.; Oh, D.H. Inactivation of bacterial pathogens on lettuce, sprouts, and spinach using hurdle technology. Innov. Food Sci. Emerg. Technol. 2017, 43, 68-76. [CrossRef]

121. Hao, J.X.; Li, J.X.; Zhao, D.D. Effect of slightly acidic electrolysed water on functional components, antioxidant and alphaglucosidase inhibitory ability of buckwheat sprouts. Int. J. Food Sci. Technol. 2021, 56, 3463-3473. [CrossRef]

122. Hao, J.X.; Wu, T.J.; Li, H.Y.; Wang, W.; Liu, H.J. Dual effects of slightly acidic electrolyzed water (SAEW) treatment on the accumulation of gamma-aminobutyric acid (GABA) and rutin in germinated buckwheat. Food Chem. 2016, 201, 87-93. [CrossRef] [PubMed]

123. Zhang, C.L.; Xia, X.D.; Li, B.M.; Hung, Y.C. Disinfection efficacy of electrolyzed oxidizing water on brown rice soaking and germination. Food Control 2018, 89, 38-45. [CrossRef]

124. Yu, Z.L.; Liu, R. Effect of electrolyzed water on enzyme activities of triticale malt during germination. J. Food Sci. Technol. 2019, 56, 1495-1501. [CrossRef] [PubMed] 
125. Qiao, W.B.; Wang, Q.F.; Han, X.; Hao, J.X. Effect of $\mathrm{pH}$ and chlorine concentration of slightly acidic electrolyzed water on the buckwheat sprouts during germination. J. Food Process. Preserv. 2019, 43, e14175. [CrossRef]

126. Li, L.Z.; Hao, J.X.; Song, S.H.; Nirasawa, S.; Jiang, Z.Q.; Liu, H.J. Effect of slightly acidic electrolyzed water on bioactive compounds and morphology of broccoli sprouts. Food Res. Int. 2018, 105, 102-109. [CrossRef] [PubMed]

127. Li, L.Z.; Song, S.H.; Nirasawa, S.; Hung, Y.C.; Jiang, Z.Q.; Liu, H.J. Slightly Acidic Electrolyzed Water Treatment Enhances the Main Bioactive Phytochemicals Content in Broccoli Sprouts via Changing Metabolism. J. Agric. Food Chem. 2019, 67, 606-614. [CrossRef]

128. Liu, R.; Yu, Z.L. Application of electrolyzed water on reducing the microbial populations on commercial mung bean sprouts. J. Food Sci. Technol. 2017, 54, 995-1001. [CrossRef] [PubMed]

129. Zhang, C.L.; Cao, W.; Hung, Y.C.; Li, B.M. Application of electrolyzed oxidizing water in production of radish sprouts to reduce natural microbiota. Food Control 2016, 67, 177-182. [CrossRef]

130. Li, X.; Hao, J.; Liu, X.; Liu, H.; Ning, Y.; Cheng, R.; Tan, B.; Jia, Y. Effect of the treatment by slightly acidic electrolyzed water on the accumulation of $\gamma$-aminobutyric acid in germinated brown millet. Food Chem. 2015, 186, 249-255. [CrossRef] [PubMed]

131. Liu, R.; Hao, J.X.; Liu, H.J.; Li, L.T. Application of electrolyzed functional water on producing mung bean sprouts. Food Control 2011, 22, 1311-1315. [CrossRef]

132. Cao, W.; Zhang, C.; Li, B. Effect of spraying subacidic electrolyzed water on buckwheat sprouts growth. Trans. Chin. Soc. Agric. Eng. 2012, 28, 159-164.

133. Liu, R.; He, X.; Shi, J.; Nirasawa, S.; Tatsumi, E.; Li, L.; Liu, H. The effect of electrolyzed water on decontamination, germination and $\gamma$-aminobutyric acid accumulation of brown rice. Food Control 2013, 33, 1-5. [CrossRef]

134. Wojdylo, A.; Nowicka, P.; Tkacz, K.; Turkiewicz, I.P. Sprouts vs. Microgreens as Novel Functional Foods: Variation of Nutritional and Phytochemical Profiles and Their In vitro Bioactive Properties. Molecules 2020, 25, 4648. [CrossRef]

135. Marton, M.; Mandoki, Z.S.; Csapo-Kiss, Z.S.; Csapo, J. The role of sprouts in human nutrition. A review. Acta Univ. Sapientiae 2010, 3, 81-117.

136. Abellan, A.; Dominguez-Perles, R.; Moreno, D.A.; Garcia-Viguera, C. Sorting out the Value of Cruciferous Sprouts as Sources of Bioactive Compounds for Nutrition and Health. Nutrients 2019, 11, 429. [CrossRef] [PubMed]

137. Kumar, I.; Sharma, R.K. Production of secondary metabolites in plants under abiotic stress: An overview. Significances Bioeng. Biosci. 2018, 2, 196-200. [CrossRef] 